\title{
Observational and energetics constraints on the non-conservation of potential/Conservative Temperature and implications for ocean modelling
}

Article

Accepted Version

Creative Commons: Attribution-Noncommercial-No Derivative Works 4.0

Tailleux, R. (2015) Observational and energetics constraints on the non-conservation of potential/Conservative Temperature and implications for ocean modelling. Ocean Modelling, 88. pp. 26-37. ISSN 1463-5003 doi: https://doi.org/10.1016/j.ocemod.2015.02.001 Available at https://centaur.reading.ac.uk/39239/

It is advisable to refer to the publisher's version if you intend to cite from the work. See Guidance on citing.

To link to this article DOI: http://dx.doi.org/10.1016/j.ocemod.2015.02.001

Publisher: Elsevier

All outputs in CentAUR are protected by Intellectual Property Rights law, including copyright law. Copyright and IPR is retained by the creators or other copyright holders. Terms and conditions for use of this material are defined in the End User Agreement. 


\section{CentAUR}

Central Archive at the University of Reading

Reading's research outputs online 


\title{
Observational and energetics constraints on the non-conservation of potential/Conservative Temperature and implications for ocean modelling
}

\author{
Rémi Tailleux \\ Department of Meteorology, University of Reading, Earley Gate, PO Box 243, Reading, \\ RG6 6BB, United Kingdom
}

\begin{abstract}
This paper seeks to elucidate the fundamental differences between the nonconservation of potential temperature and that of Conservative Temperature, in order to better understand the relative merits of each quantity for use as the heat variable in numerical ocean models. The main result is that potential temperature is found to behave similarly to entropy, in the sense that its nonconservation primarily reflects production/destruction by surface heat and freshwater fluxes; in contrast, the nonconservation of Conservative Temperature is found to reflect primarily the overall compressible work of expansion/contraction. This paper then shows how this can be exploited to constrain the nonconservation of potential temperature and entropy from observed surface heat fluxes, and the nonconservation of Conservative Temperature from published estimates of the mechanical energy budgets of ocean numerical models. Finally, the paper shows how to modify the evolution equation for potential temperature so that it is exactly equivalent to using an exactly conservative evolution equation for Conservative Temperature, as was recently recommended by IOC et al. (2010). This result should in
\end{abstract}


principle allow ocean modellers to test the equivalence between the two formulations, and to indirectly investigate to what extent the budget of derived nonconservative quantities such as buoyancy and entropy can be expected to be accurately represented in ocean models.

Keywords:

ocean modelling, conservation equations, heat non-conservation, energy conservation, potential temperature, Conservative Temperature

\section{Introduction}

The issue of whether potential temperature $\theta$ is the most appropriate heat variable to be used in numerical ocean general circulation models (OGCMs) has recently come under scrutiny following McDougall (2003) and IOC et al. (2010) suggestion that $\theta$ should be replaced by Conservative Temperature (CT or $\Theta$ ). The main argument, originally made by McDougall (2003), is that the current practice of treating $\theta$ as a conservative quantity is significantly inaccurate, and to the extent that one should insist in treating heat as conservative in OGCMs, it appears to be significantly more accurate to do so by using CT instead. Since CT is a relatively new quantity, its formal properties have yet to be fully understood, so that the full implications of switching from $\theta$ to CT in OGCMs are not all entirely clear. The alternative option - to accept that potential temperature is fundamentally nonconservative and to modify its model formulation accordingly — has not received attention so far, but needs to be understood to inform the debate about whether to switch or not. The main purpose of this paper is to achieve a deeper understanding of the fundamental differences between the nonconser- 
vation of potential temperature and that of Conservative Temperature, in order to help ocean modellers better understand the pros and cons of each modelling choice.

From a fundamental viewpoint, the nonconservation of heat (arising from irreversible processes) as measured by potential temperature, Conservative Temperature or entropy is now well understood to be a natural consequence of energy conservation, in the sense that were heat to be conservative, total energy would be nonconservative and conversely, as shown in Tailleux (2010a). Tailleux (2010a) proposed to extend this idea to coarse-grained primitive hydrostatic Boussinesq models as a practical way (and somewhat ad-hoc) to evaluating the nonconservation of $\theta$ and CT in such models. Specifically, the method works as follows. Starting from the hydrostatic Boussinesq primitive equations formulated in terms of either potential temperature, Conservative Temperature or entropy, one writes down the evolution equation for the total energy assuming that the evolution equation for any of the heat variables is the sum of a conservative part (expressed as the divergence of some flux) plus an a priori unknown nonconservative part. Both conservative and nonconservative terms appear in the equation for total energy thus obtained. As discussed by Tailleux (2010a), each nonconservative term is associated with some inconsistency in the model formulation, such as using the total hydrostatic pressure instead of the Boussinesq pressure in the equation of state for instance. One of the nonconservative terms in the total energy equation is directly related to the heat nonconservation term. Imposing such a term to be zero, as required by the principle of energy conservation, provides an explicit expression for the heat nonconservative term in terms of the heat 
and salt fluxes, which depends on the assumed form of the turbulent ocean mixing processes. Graham and McDougall (2013) uses a similar approach to quantify heat nonconservation, but which relies on the existence of a different conservative quantity (a locally referenced potential enthalpy) than total energy. Their approach yields a different expression for the nonconservative terms than that of Tailleux (2010a), which among other things, lacks pressure gradient terms. Although their approaches rely on different assumptions, it is important to point out that Tailleux (2010a) and Graham and McDougall (2013) nevertheless agree that the expression for the nonconservation of $\theta$ and $\Theta$ in terms of the turbulent fluxes follows directly from the particular quantity that one assumes to be conservative for the averaged equations of motion. As an alternative to Tailleux (2010a), who assumed the latter to be total energy, and to Graham and McDougall (2013), who assumed it to be a locally defined form of potential enthalpy, one may simply assume CT to be exactly conservative, as recommended by IOC et al. (2010), and derive the implied form for the nonconservation of $\theta$. Such an idea will be exploited in Section 5 .

The main objective of this paper is to clarify the nature of the nonconservation of potential/Conservative Temperature and of entropy. Building upon the results by Tailleux (2012), we argue that the nonconservative production of Conservative Temperature or potential enthalpy fundamentally measures the thermodynamic work of expansion/contraction. In other words, in the same way that McDougall (2003) argues that potential enthalpy is the most appropriate variable to measure "heat" into the oceans, we argue that the non-conservation of potential enthalpy measures the "work" done 
by compressible effects. We also argue that the nonconservative production of potential temperature is of a fundamentally different nature, and actually measures the production of potential temperature by surface heat fluxes (and to a lesser extent freshwater fluxes), in the same way that irreversible entropy production reflects the entropy production by surface fluxes in a steady-state system.

This paper is organised as follows. Section 2 recalls the general construction of the nonconservative production terms and their link to energy conservation initiated in Tailleux (2010a) and Tailleux (2012), and further shows how to link the non conservation of potential temperature and entropy to ocean surface properties; such a link is well known for entropy, but not for potential temperature. Section 3 discusses a priori estimates for the non conservation terms, as well as some of their theoretical properties. Section 4 uses observations to illustrate and quantify empirically the results of Section 3. Section 5 offers a summary and discussion of the implications of our results for ocean modelling, which leads us to propose a modification of the evolution equation for $\theta$ that is meant to be equivalent to a strictly conservative evolution equation for $\Theta$ and hence that we propose as a basis for informing the debate about switching or not.

\section{Non-conservation of "heat" variables for the fully compressible Navier-Stokes equations for seawater \\ 2.1. Compressible Navier-Stokes equations and "heat" variables \\ The nonconservativeness of different measures of heat is discussed in the context of the full compressible Navier-Stokes equations (NCS) in a rotating}


92

93

94

$$
\rho \frac{D \mathbf{v}}{D t}+2 \Omega \times(\rho \mathbf{v})+\nabla P=-\rho \nabla \Phi+\rho \nabla \cdot \mathbf{S}
$$

$$
\frac{D \rho}{D t}+\rho \nabla \cdot \mathbf{v}=0
$$

$$
\rho \frac{D S}{D t}=-\nabla \cdot\left(\rho \mathbf{F}_{S}\right)
$$

95

110

111

where $\mathbf{v}=(u, v, w)$ is the three-dimensional velocity field, $D / D t=\partial_{t}+\mathbf{v} \cdot \nabla$ is the substantial derivative, $P$ is the pressure, $\rho$ is the density, $\Phi=g_{0} Z$ is the geopotential, $g_{0}$ is the acceleration of gravity, $\Omega$ is Earth's rotation vector, $\mathbf{S}$ is the stress tensor, $\Phi=g_{0} Z$ is the geopotential formulated in terms of a constant gravitational potential acceleration $g_{0}$ and geometric height $Z=z$, with $z$ the regular height increasing upwards. Chemical composition is described by the salinity $S$ (which in practice one may assume to be the Absolute Salinity defined in IOC et al. (2010)), and $\mathbf{F}_{S}$ is the diffusive salinity flux.

\subsection{Heat variables}

As in Graham and McDougall (2013), we focus on the classical measures of heat based on specific entropy $\eta$ and potential temperature $\theta$, as well as on the more recent Conservative Temperature $\Theta$. As these variables are all nonconservative, they can all a priori be assumed to satisfy evolution equations of the form

$$
\rho \frac{D \eta}{D t}=-\nabla \cdot\left(\rho \mathbf{F}_{\eta}\right)+\rho \dot{\eta}_{i r r}
$$

$$
\rho \frac{D \theta}{D t}=-\nabla \cdot\left(\rho \mathbf{F}_{\theta}\right)+\rho \dot{\theta}_{i r r}
$$

$$
\rho \frac{D \Theta}{D t}=-\nabla \cdot\left(\rho \mathbf{F}_{\Theta}\right)+\rho \dot{\Theta}_{i r r}
$$


where $\mathbf{F}_{\eta}, \mathbf{F}_{\Theta}$, and $\mathbf{F}_{\theta}$ are the fluxes of each quantity, involving radiative effects, molecular diffusion, and latent heat release, while $\dot{\eta}_{i r r}, \dot{\theta}_{i r r}$ and $\dot{\Theta}_{i r r}$ are the non-conservation terms for each quantity that are the main focus of the present paper.

Physically, potential temperature $\theta$ represents the temperature that a parcel would have if brought to the surface adiabatically at constant composition, and is therefore fundamentally linked to entropy, being implicitly defined by the relation

$$
\eta\left(\theta, S, P_{0}\right)=\eta(T, S, P)
$$

where $P_{0}$ is a reference mean atmospheric pressure, and $T$ the in-situ temperature. Note that throughout the manuscript, both $\theta$ and $T$ denote absolute temperatures expressed in kelvin, as some of the quantities discussed below, such as the ratio $T / \theta$ would not make sense if $T$ and $\theta$ were expressed on the Celsius scale. In contrast, Conservative Temperature is defined as being proportional to potential enthalpy $h_{\theta}$, such that $c_{p}^{0} \Theta=h_{\theta}$, with the potential enthalpy being defined as the enthalpy that a parcel would have if brought adiabatically to the surface, and thus implicitly defined by the relation

$$
h_{\theta}=h\left(\eta, S, P_{0}\right),
$$

or equivalently as $\eta(h, S, P)=\eta\left(h_{\theta}, S, P_{0}\right)$, where $c_{p}^{0}$ is a constant defined in McDougall (2003) and IOC et al. (2010).

\subsection{Passage relations for "heat" variables}

How entropy, potential temperature and Conservative Temperature are related to each other has been previously discussed in Tailleux (2010a) building upon previous work by Bacon and Fofonoff (1996) and McDougall (2003) 
(see also IOC et al. (2010)). All the necessary relations are usually obtained from the total differential of the specific enthalpy $h$ (also often referred to as the fundamental relation of thermodynamics), viz.

$$
\mathrm{d} h=T \mathrm{~d} \eta+\mu \mathrm{d} S+v \mathrm{~d} P
$$

e.g., IOC et al. (2010), which can alternatively be written in terms of temperature, salinity and pressure as follows:

$$
\mathrm{d} h=c_{p} \mathrm{~d} T+\left(\mu-T \frac{\partial \mu}{\partial T}\right) \mathrm{d} S+v(1-\alpha T) \mathrm{d} P,
$$

where $c_{p}$ is the specific heat capacity at constant pressure, $\mu$ is the relative chemical potential of seawater, $v=1 / \rho$ is the specific volume and $\alpha$ is the thermal expansion coefficient. The passage from (9) to (10) follows from the fact that the total differential of specific entropy in terms of temperature, salinity and pressure is given by:

$$
\mathrm{d} \eta=\frac{c_{p}}{T} \mathrm{~d} T-\frac{\partial \mu}{\partial T} \mathrm{~d} S-\frac{\alpha}{\rho} \mathrm{d} P
$$

e.g., Tailleux (2010a). How the term $T \mathrm{~d} \eta+\mu \mathrm{d} S$ in the enthalpy differential (9) transforms in the $(\theta, S)$ and $(\Theta, S)$ representations is given by Eqs. (B.2) and (B.3) of Tailleux (2010a) and in Appendix A.12 of IOC et al. (2010), specifically

$$
\mu \mathrm{d} S+T \mathrm{~d} \eta=\left(\mu-T \frac{\partial \mu_{R}}{\partial \theta}\right) \mathrm{d} S+\frac{T c_{p}^{R}}{\theta} \mathrm{d} \theta
$$

$$
\mu \mathrm{d} S+T \mathrm{~d} \eta=\left(\mu-\frac{T \mu_{R}}{\theta}\right) \mathrm{d} S+\frac{T c_{p}^{0}}{\theta} \mathrm{d} \Theta,
$$

where $\mu_{R}=\mu\left(\theta, S, P_{0}\right)$ and $c_{p}^{R}=c_{p}\left(\theta, S, P_{0}\right)$. These relations were first derived by Bacon and Fofonoff (1996) and McDougall (2003) respectively. 
Also useful are relations allowing to pass from the $(\theta, S)$ to $(\Theta, S)$ representation, which can be obtained by eliminating $\eta$ between (12) and (13), which yields:

$$
\begin{aligned}
\mathrm{d} \Theta & =\frac{1}{c_{p}^{0}}\left(\mu_{R}-\theta \frac{\partial \mu_{R}}{\partial \theta}\right) \mathrm{d} S+\frac{c_{p}^{R}}{c_{p}^{0}} \mathrm{~d} \theta, \\
\mathrm{d} \theta & =\frac{c_{p}^{0}}{c_{p}^{R}} \mathrm{~d} \Theta-\frac{1}{c_{p}^{R}}\left(\mu_{R}-\theta \frac{\partial \mu_{R}}{\partial \theta}\right) \mathrm{d} S .
\end{aligned}
$$

These two equations correspond to Eqs. (A.14) and (A.15) in Tailleux (2010a).

\subsection{Implications for the evolution equations of the heat variables}

The simplest way to obtain explicit expressions for the fluxes and nonconservative terms entering the evolution equations for entropy, potential temperature and Conservative Temperature (4)-(6) is to deduce the latter from the evolution equation for enthalpy, which energy considerations impose to be of the following form

$$
\rho \frac{D h}{D t}=-\nabla \cdot[\rho \underbrace{\left(\mathbf{F}_{h}+\mathbf{F}_{r a d}+\mathbf{F}_{o a}\right)}_{\mathbf{F}_{h t o t}}]+\rho \varepsilon_{K}+\frac{D P}{D t} .
$$

In the above, $\mathbf{F}_{h}$ represents the enthalpy flux due to the molecular diffusive fluxes of heat and salt, $\mathbf{F}_{\text {rad }}$ represents represent the enthalpy flux due to incoming shortwave radiation and outgoing/downwelling long wave radiation, and $\mathbf{F}_{o a}$ is used to represent the decrease in ocean enthalpy following latent heat release associated with evaporation. Eq. (16) is similar to that given in IOC et al. (2010), except for the term $\mathbf{F}_{o a}$. By comparing (16) with the expression for the total derivative of enthalpy, viz.,

$$
\frac{D h}{D t}=T \frac{D \eta}{D t}+\mu \frac{D S}{D t}+v \frac{D P}{D t}
$$


171

it follows that

$$
\rho\left(T \frac{D \eta}{D t}+\mu \frac{D S}{D t}\right)=-\nabla \cdot\left[\rho\left(\mathbf{F}_{h}+\mathbf{F}_{r a d}+\mathbf{F}_{o a}\right)\right]+\rho \varepsilon_{K},
$$

181

where $\varepsilon_{K}$ is the viscous dissipation rate, which is related to the work against the stress tensor in the classical way, e.g., see Landau and Lifschitz (1987); Tailleux (2010b). Now, by combining (18) with the passage relations derived above and Eq. (3) for salinity, it is easy using elementary manipulations to obtain the results presented in the following paragraphs.

Flux and non conservation of entropy. First, (18) shows that the evolution equation for entropy can be written as

$$
\rho \frac{D \eta}{D t}=-\frac{\nabla \cdot\left(\rho \mathbf{F}_{h t o t}\right)}{T}+\frac{\mu \nabla \cdot\left(\rho \mathbf{F}_{S}\right)}{T}+\frac{\rho \varepsilon_{K}}{T} .
$$

This can be written in the generic form

$$
\rho \frac{D \eta}{D t}=-\nabla \cdot\left(\rho \mathbf{F}_{\eta}\right)+\rho \dot{\eta}_{i r r}
$$

provided that $\mathbf{F}_{\eta}$ and $\dot{\eta}_{i r r}$ are given by

$$
\mathbf{F}_{\eta}=\frac{\mathbf{F}_{h t o t}-\mu \mathbf{F}_{S}}{T}
$$

$$
\dot{\eta}_{i r r}=-\mathbf{F}_{S} \cdot \nabla\left(\frac{\mu}{T}\right)+\mathbf{F}_{h t o t} \cdot \nabla\left(\frac{1}{T}\right)+\frac{\varepsilon_{K}}{T} .
$$

Flux and non conservation of Conservative Temperature. By using (13) and (18), it follows that we have

$$
\rho\left[\frac{T c_{p}^{0}}{\theta} \frac{D \Theta}{D t}+\left(\mu-\frac{T \mu_{R}}{\theta}\right) \frac{D S}{D t}\right]=-\nabla \cdot\left(\rho \mathbf{F}_{h t o t}\right)+\rho \varepsilon_{K} .
$$

After some manipulation, it is possible to rewrite this equation in the generic form

$$
\rho \frac{D \Theta}{D t}=-\nabla \cdot\left(\rho \mathbf{F}_{\Theta}\right)+\rho \dot{\Theta}_{i r r}
$$


186

187

$$
\dot{\Theta}_{i r r}=\frac{\theta}{T c_{p}^{0}}\left[-\mathbf{F}_{S} \cdot \nabla\left(\mu-\frac{T \mu_{R}}{\theta}\right)-\mathbf{F}_{\Theta} \cdot \nabla\left(\frac{T c_{p}^{0}}{\theta}\right)+\varepsilon_{K}\right] .
$$

188 189

193

$$
\dot{\theta}_{i r r}=\frac{\theta}{T c_{p}^{R}}\left[-\mathbf{F}_{S} \cdot \nabla\left(\mu-T \frac{\partial \mu_{R}}{\partial \theta}\right)-\mathbf{F}_{\theta} \cdot \nabla\left(\frac{T c_{p}^{R}}{\theta}\right)+\varepsilon_{K}\right] .
$$

194

197

198

Flux and non conservation of potential temperature. By using (12) and (18), it follows that we have

$$
\rho\left[\frac{T c_{p}^{R}}{\theta} \frac{D \theta}{D t}+\left(\mu-T \frac{\partial \mu_{R}}{\partial \theta}\right) \frac{D S}{D t}\right]=-\nabla \cdot\left(\rho \mathbf{F}_{h t o t}\right)+\rho \varepsilon_{K} .
$$

After some manipulation, it is possible to rewrite this equation in the generic form

$$
\rho \frac{D \theta}{D t}=-\nabla \cdot\left(\rho \mathbf{F}_{\theta}\right)+\rho \dot{\theta}_{i r r}
$$

192 provided that $\mathbf{F}_{\theta}$ and $\dot{\theta}_{i r r}$ are given by

$$
\mathbf{F}_{\theta}=\frac{\theta}{c_{p}^{R} T}\left[\mathbf{F}_{h t o t}-\left(\mu-T \frac{\partial \mu_{R}}{\partial \theta}\right) \mathbf{F}_{S}\right]
$$

form of the fluxes of $\theta$ and $\Theta$ at the surface. Evaluating (25) and (29) at $z=0$ yields respectively:

$$
\mathbf{F}_{\Theta}=\frac{1}{c_{p}^{0}}\left(\mathbf{F}_{h}+\mathbf{F}_{r a d}+\mathbf{F}_{o a}\right), \quad \text { at } \quad \mathrm{z}=0,
$$

$$
\mathbf{F}_{\theta}=\frac{1}{c_{p}^{R}}\left(\mathbf{F}_{q}+\mathbf{F}_{\text {rad }}+\mathbf{F}_{o a}\right), \quad \text { at } \quad \mathrm{z}=0,
$$

where

$$
\mathbf{F}_{q}=\mathbf{F}_{h}-\left(\mu-T \frac{\partial \mu}{\partial T}\right) \mathbf{F}_{S}
$$


is a reduced heat flux that is discussed extensively below, by noting that $\mu=\mu_{R}$ at $z=0$. The implications for the appropriate boundary conditions for $\theta$ and $\Theta$ are discussed in Section 5.

\section{Linking heat non-conservation to ocean surface properties}

An important difficulty with the form of the nonconservative terms for potential temperature, Conservative Temperature and entropy derived by Tailleux (2010a) and Graham and McDougall (2013) is that they rely on using explicit turbulent mixing parameterisations, which remain poorly constrained. The purpose of this section is to show that $\dot{\eta}_{i r r}, \dot{\theta}_{i r r}$ and $\dot{\Theta}_{i r r}$ can be related to measurable or derived properties provided that the oceans can be regarded as being in quasi steady state, which provides an independent mean to estimate such terms. To that end, it is important to first review the formulation of boundary conditions for heat, salt and freshwater for the ocean under the most general conditions.

\subsection{Boundary conditions for salt and freshwater}

The standard formulation of boundary conditions for salt and freshwater assumes that the latter move with different velocities $\mathbf{v}_{s}$ and $\mathbf{v}_{w}$ respectively, and that each satisfies a conservation equation of the form

$$
\frac{\partial \rho_{s}}{\partial t}+\nabla \cdot\left(\rho \mathbf{v}_{s}\right)=0
$$

$$
\frac{\partial \rho_{w}}{\partial t}+\nabla \cdot\left(\rho \mathbf{v}_{w}\right)=0
$$

where $\rho_{s}=\rho S$ and $\rho_{w}=\rho(1-S)$ are the partial densities for salt and freshwater respectively, e.g., Warren (2006). According to non-equilibrium 
230

thermodynamics, molecular diffusion of salt in solution arises from the difference between $\mathbf{v}_{w}$ and $\mathbf{v}_{s}$. The effect can be isolated by introducing the barycentric velocity $\mathbf{v}=S \mathbf{v}_{s}+(1-S) \mathbf{v}_{w}$, which allows one to rewrite the above conservations equations as follows

$$
\frac{\partial \rho_{s}}{\partial t}+\nabla \cdot\left(\rho_{s} \mathbf{v}\right)=-\nabla \cdot\left(\rho \mathbf{F}_{S}\right), \quad \frac{\partial \rho_{w}}{\partial t}+\nabla \cdot\left(\rho_{w} \mathbf{v}\right)=\nabla \cdot\left(\rho \mathbf{F}_{S}\right)
$$

where the salt flux $\mathbf{F}_{S}$ is defined by

$$
\rho \mathbf{F}_{S}=\rho_{s}\left(\mathbf{v}_{s}-\mathbf{v}\right)=\rho S(1-S)\left(\mathbf{v}_{s}-\mathbf{v}_{w}\right),
$$

which establishes that salt flux is only nonzero when $\mathbf{v}_{s}$ and $\mathbf{v}_{w}$ are different. The boundary conditions for $\mathbf{v}_{s}$ and $\mathbf{v}_{w}$ have been discussed by several authors, e.g., Warren (2006), IOC et al. (2010); if one assumes the sea surface height to be given by an equation of the form $z=\zeta(x, y, t)$ (which neglects spray and gravity waves overturns), they take the form

$$
\frac{\partial \zeta}{\partial t}+\mathbf{u}_{s} \cdot \nabla_{h} \zeta-w_{s}=0
$$

$$
\rho_{w}\left[\frac{\partial \zeta}{\partial t}+\mathbf{u}_{w} \cdot \nabla_{h} \zeta-w_{w}\right]=\rho_{f}(P-E),
$$

where $\rho_{w}=\rho(T, S, p)(1-S)$ is the partial density of freshwater in seawater, while $\rho_{f}=\rho(T, 0, p)$ is the density of freshwater. Physically, the condition for salt assumes that no salt leaves the ocean, which is an idealisation, while that for freshwater assumes that the latter enters and leaves the ocean through precipitation $P$ and evaporation $E$. Also useful is the boundary condition for the velocity difference $\mathbf{v}_{s}-\mathbf{v}_{w}$, obtained by taking the difference between (38) and (39),

$$
\left(\mathbf{u}_{s}-\mathbf{u}_{w}\right) \cdot \nabla_{h} \zeta-\left(w_{s}-w_{w}\right)=\frac{\rho_{f}(P-E)}{\rho_{w}}
$$


which in turn implies the following boundary condition for the salt flux

$$
\rho \mathbf{F}_{S} \cdot \mathbf{n} \mathrm{d} \Sigma=\rho S(1-S)\left(\mathbf{v}_{s}-\mathbf{v}_{w}\right) \cdot \mathbf{n d} \Sigma=-\rho_{f} S(E-P) \mathrm{d} A,
$$

where $\mathrm{d} A=\mathrm{d} x \mathrm{~d} y$ is the flat areal surface element, while $\mathrm{d} \Sigma=\sqrt{1+\left\|\nabla_{h} \zeta\right\|^{2}} \mathrm{~d} A$ is the elemental area normal to the outward unit vector $\mathbf{n}$, e.g., see BeronVera et al. (1999).

\subsection{Boundary conditions for heat}

The formulation of the boundary conditions for the surface enthalpy flux due to radiation and latent heat release is straightforward, and given by

$$
-\rho \mathbf{F}_{r a d} \cdot \mathbf{n} \mathrm{d} \Sigma=\left(Q_{s w}+Q_{l w}\right) \mathrm{d} A
$$

$$
-\rho \mathbf{F}_{o a} \cdot \mathbf{n} \mathrm{d} \Sigma=Q_{l h} \mathrm{~d} A
$$

where $Q_{s w}$ is the incoming shortwave radiation, $Q_{l w}$ is the sum of the outgoing and incoming downwelling long wave radiation, and $Q_{l h}=-L E<0$ is the latent heat flux, where $L=h_{v}-h_{w}$ is the latent heat flux, defined as the difference between the partial enthalpy of water vapour in moist air minus the partial enthalpy of freshwater in seawater, e.g., see Eq. (3.39.7) of IOC et al. (2010).

The proper formulation of the boundary condition for the diffusive flux of enthalpy $\mathbf{F}_{h}$ requires some care, as the latter is a priori affected by both salt and heat diffusion, but only the thermal part is related to the sensible heat flux. The way to remove the effects of salt diffusion can be achieved by introducing the reduced heat flux $\mathbf{F}_{q}$ that captures the effects of heat diffusion alone (assuming cross diffusive effects such as the Soret and Dufour 
effects can be neglected), defined by

$$
\mathbf{F}_{q}=\mathbf{F}_{h}-\left.\frac{\partial h}{\partial S}\right|_{T, P} \mathbf{F}_{S}=\mathbf{F}_{h}-\left(\mu-T \frac{\partial \mu}{\partial T}\right) \mathbf{F}_{S} \approx-\kappa c_{p} \nabla T,
$$

where $\kappa$ is the molecular diffusion of heat, e.g., Landau and Lifschitz (1987). It is therefore $\mathbf{F}_{q}$, rather than $\mathbf{F}_{h}$, whose boundary condition is related to the sensible heat flux, viz,

$$
-\rho \mathbf{F}_{q} \cdot \mathbf{n} \mathrm{d} \Sigma=Q_{\text {sens }} \mathrm{d} A,
$$

where $Q_{\text {sens }}$ is the sensible heat flux.

\subsection{Remarks on the conservative form of heat evolution equations}

The study of volume-integrated budgets if facilitated by writing down evolution equations in conservative form, which usually result from combining the advective form of the equations with the mass conservation equation, which is illustrated by the transformation

$$
\rho \frac{D q}{D t} \quad \rightarrow \quad \frac{\partial(\rho q)}{\partial t}+\nabla \cdot(\rho q \mathbf{v})
$$

We argue, however, that the above conservative form (46) for the mass flux of $q$ is not optimal, since boundary conditions are more naturally formulated for the salt and freshwater velocities $\mathbf{v}_{s}$ and $\mathbf{v}_{w}$ rather than for the barycentric velocity $\mathbf{v}$; moreover, a process such as evaporation distillates the salt from the freshwater part of seawater, which motivates us to write $q$ in terms of its partial salt and freshwater parts $q_{s}$ and $q_{w}$ as $q=S q_{s}+(1-S) q_{w}$, where $q_{s}$ and $q_{w}$ are defined by

$$
q_{s}=q+(1-S) \frac{\partial q}{\partial S}, \quad q_{w}=q-S \frac{\partial q}{\partial S}
$$


e.g., IOC et al. (2010). This in turn implies

$$
q_{s}-q_{w}=\frac{\partial q}{\partial S}
$$

where the partial derivative with respect to salinity is done at constant temperature and pressure. Making use of the above, as well as of the definition for the salt flux $\mathbf{F}_{S}=S(1-S)\left(\mathbf{v}_{s}-\mathbf{v}_{w}\right)$, allows one to rewrite the advective enthalpy flux as follows

$$
\begin{gathered}
h \mathbf{v}=\left[S h_{s}+(1-S) h_{w}\right] \mathbf{v}= \\
=S h_{s} \mathbf{v}_{s}+(1-S) h_{w} \mathbf{v}_{w}+S h_{s}\left(\mathbf{v}-\mathbf{v}_{s}\right)+(1-S) h_{w}\left(\mathbf{v}-\mathbf{v}_{w}\right) \\
=S h_{s} \mathbf{v}_{s}+(1-S) h_{w} \mathbf{v}_{w}-\frac{\partial h}{\partial S} \mathbf{F}_{S} .
\end{gathered}
$$

Using a similar approach allows one to rewrite the advective entropy flux as follows

$$
\eta \mathbf{v}=S \eta_{s} \mathbf{v}_{s}+(1-S) h_{w} \mathbf{v}_{w}-\frac{\partial \eta}{\partial S} \mathbf{F}_{S}
$$

The main advantage of (49) and (50) is to elucidate the fact that the classical advective fluxes of enthalpy and entropy are actually made up of both mass and diffusive fluxes, which is not a priori obvious and rarely discussed (Warren (2006) alludes to it, but not very clearly). Next, we make use of standard thermodynamic relations to link $\partial h / \partial S$ and $\partial \eta / \partial S$ to the relative chemical potential $\mu$ and its temperature derivative as follows

$$
\left.\frac{\partial h}{\partial S}\right|_{T, P}=\mu-T \frac{\partial \mu}{\partial T},\left.\quad \frac{\partial \eta}{\partial S}\right|_{T, P}=-\frac{\partial \mu}{\partial T},
$$

which we then use to rewrite the sum of the advective and diffusive fluxes of enthalpy and entropy as follows

$$
h \mathbf{v}+\mathbf{F}_{h}=S h_{s} \mathbf{v}_{s}+(1-S) h_{w} \mathbf{v}_{w}+\mathbf{F}_{q}
$$


288

294

302

303

$$
\eta \mathbf{v}+\frac{\mathbf{F}_{h}-\mu \mathbf{F}_{S}}{T}=S \eta_{s} \mathbf{v}_{s}+(1-S) \eta_{w} \mathbf{v}_{w}+\frac{\mathbf{F}_{q}}{T}
$$

These relations are important and more useful, because they more clearly link the advective and diffusive parts of the fluxes to their boundary conditions by removing salt diffusion effects entirely. As a result, it is possible to rewrite the conservative form of the enthalpy and entropy evolution equations as follows

$$
\frac{\partial(\rho \eta)}{\partial t}+\nabla \cdot\left[\rho_{s} \eta_{s} \mathbf{v}_{s}+\rho_{w} \eta_{w} \mathbf{v}_{w}\right]+\nabla \cdot\left[\rho\left(\frac{\mathbf{F}_{q}+\mathbf{F}_{r a d}+\mathbf{F}_{o a}}{T}\right)\right]=\rho \dot{\eta}_{i r r}
$$

$$
\frac{\partial(\rho h)}{\partial t}+\nabla \cdot\left[\rho_{s} h_{s} \mathbf{v}_{s}+\rho_{w} h_{w} \mathbf{v}_{w}\right]+\nabla \cdot\left[\rho\left(\mathbf{F}_{q}+\mathbf{F}_{r a d}+\mathbf{F}_{o a}\right)\right]=\rho \varepsilon_{K}+\frac{D P}{D t}
$$

which, as discussed below, greatly facilitates the understanding of the global budgets of the heat variables.

\subsection{Insights from global budgets}

Having clarified the nature of the boundary conditions for freshwater, salt, and heat, it is straightforward to show that the temporal evolution of the volume-integrated entropy, Conservative Temperature and potential temperature must be given by

$$
\frac{d}{d t} \int_{V} \rho \eta \mathrm{d} V=\int_{S} \frac{Q_{n e t}}{T} \mathrm{~d} A+\int_{V} \rho \dot{\eta}_{i r r}+\int_{S} \rho_{f} \eta_{w}(P-E) \mathrm{d} A,
$$

$$
\frac{d}{d t} \int_{V} \rho \Theta \mathrm{d} V=\int_{S} \frac{Q_{n e t}}{c_{p}^{0}}+\int_{V} \rho \dot{\Theta}_{i r r} \mathrm{~d} V+\int_{S} \frac{\rho_{f} h_{w}(P-E)}{c_{p}^{0}} \mathrm{~d} A,
$$

$$
\frac{d}{d t} \int_{V} \rho \theta \mathrm{d} V=\int_{S} \frac{Q_{n e t}}{c_{p}^{R}} \mathrm{~d} A+\int_{V} \rho \dot{\theta}_{i r r} \mathrm{~d} V+\int_{S} \rho_{f} T_{s}(P-E) \mathrm{d} A,
$$

where $T_{s}$ is the ocean surface temperature, $Q_{n e t}=Q_{\text {sens }}+Q_{s h}+Q_{l w}+Q_{l h}$ is the sum of all heat flux components. Note also that the symbol $S$ in the 
315

316

integral refers to a surface integral and is not to be confused with salinity as in most of the paper. These expressions show that the volume integral of each quantity involves a term related to the net downward heat flux $Q_{n e t}$, a nonconservative term related to irreversible diffusive and viscous effects, and a mass flux term related to evaporation and precipitation (run-off is assumed to be included into precipitation, and will not be explicitly mentioned again). By considering a sufficiently long time average of the above budgets that approximately statistically steady-state conditions can be assumed to hold, the following constraints on the nonconservative terms are obtained

$$
\int_{V} \rho \dot{\eta}_{i r r} \mathrm{~d} V \approx-\int_{S} \frac{Q_{n e t}}{T} \mathrm{~d} A-\int_{S} \rho_{f} \eta_{w}(P-E) \mathrm{d} A
$$
6

$$
\begin{aligned}
\int_{V} \rho \dot{\Theta}_{i r r} \mathrm{~d} V & \approx-\int_{S} \frac{Q_{n e t}}{c_{p}^{0}} \mathrm{~d} A-\int_{S} \frac{\rho_{f} h_{w}(P-E)}{c_{p}^{0}} \mathrm{~d} A \\
\int_{V} \rho \dot{\theta}_{i r r} \mathrm{~d} V & \approx-\int_{S} \frac{Q_{n e t}}{c_{p}^{R}} \mathrm{~d} A+\int_{S} \rho_{f} T_{s}(P-E) \mathrm{d} A .
\end{aligned}
$$

Assuming that precipation (including run-off) balances evaporation globally, each of the term involving $(P-E)$ can be written in the form

$$
\int_{S} \rho_{f} Q(P-E) \mathrm{d} A \approx \Delta Q M_{E}
$$

where $M_{E}$ is the total mass flux due to either precipitation or evaporation (assuming the two balance) in $\mathrm{kg} / \mathrm{s}$, and $\Delta Q=Q_{P}-Q_{E}$ is the difference between a representative value of $Q$ for precipitation and $Q_{E}$ a representative value for evaporation. As mentioned in Griffies et al (2009), the term involving precipitation and evaporation is usually found to be subdominant in the heat budget, and is therefore neglected in the rest of the paper, but could be easily retained in a more rigorous analysis (although not necessarily 
easy to estimate precisely). As a result, we approximate the above integrals as follows

$$
\begin{aligned}
\int_{V} \rho \dot{\eta}_{i r r} \mathrm{~d} V & \approx-\int_{S} \frac{Q_{n e t}}{T} \mathrm{~d} S \\
\int_{V} \rho \dot{\Theta}_{i r r} \mathrm{~d} V & \approx-\int_{S} \frac{Q_{n e t}}{c_{p}^{0}} \mathrm{~d} S .
\end{aligned}
$$

$$
\int_{V} \rho \dot{\theta}_{i r r} \mathrm{~d} V \approx-\int_{S} \frac{Q_{n e t}}{c_{p}^{R}} \mathrm{~d} S
$$

Before examining the implications of the above results, it is useful to recall the constraints on the net heat flux that needs to hold for a steady-state ocean following from the global conservation of energy, as well as from the balance equation for mechanical energy. To that end, it is first useful to write the net flux $Q_{\text {total }}=\int_{S} Q_{n e t} \mathrm{~d} A=Q_{\text {in }}-Q_{\text {out }}$ as the difference between a net positive input of heat minus a net cooling term. For a steady-state ocean, $Q_{\text {out }} \neq Q_{\text {in }}$ because the existence of mechanical sources of energy (due to the wind, tides, atmospheric pressure work, ...) implies that the total energy budget is given by:

$$
Q_{\text {in }}-Q_{\text {out }}+W_{\text {mech }}=0
$$

where $W_{\text {mech }}$ denotes the power input by the mechanical sources of energy, see Tailleux (2010b) for more details on how to arrive at this result. Another useful constraint is obtained from the budget of mechanical energy (i.e., the sum of kinetic energy and gravitational potential energy), which leads to:

$$
W_{\text {mech }}+\underbrace{\int_{V} P \frac{D v}{D t} \mathrm{~d} m}_{B}=\underbrace{\int_{V} \rho \varepsilon_{K} \mathrm{~d} V}_{D(K E)},
$$

where the term $B$ represents the classical thermodynamic work of expansion/contraction, whereas $D(K E)$ denotes the total viscous dissipation. 


\subsection{A priori estimate of nonconservative production of $\Theta$}

Because the nature of its non conservation is quite different from that of entropy and potential temperature, we discuss Conservative Temperature first. By combining (64) and (66), it follows that:

$$
\int_{V} \rho \dot{\Theta}_{i r r} \mathrm{~d} V \approx-\frac{Q_{\text {total }}}{c_{p}^{0}}=\frac{Q_{\text {out }}-Q_{\text {in }}}{c_{p}^{0}}=\frac{W_{\text {mech }}}{c_{p}^{0}}>0,
$$

which states that the total nonconservative production of Conservative Temperature actually measures the overall power input due to the mechanical sources of energy, which is expected to be strictly positive. As established previously, the nonconservative production of $\Theta$ is the sum of two parts, one related to molecular diffusive processes, one related to viscous dissipation. We are primarily interested in estimating the former, which is the part primarily discussed in Tailleux (2010a) and Graham and McDougall (2013), and which we denote by $\dot{\Theta}_{\text {irr }}^{\text {diff }}$. This leads us to rewrite (68) as follows:

$$
\int_{V} \rho \dot{\Theta}_{\text {irr }}^{\text {diff }} \mathrm{d} V+\int_{V} \frac{\theta}{T c_{p}^{0}} \rho \varepsilon_{K} \mathrm{~d} V=\frac{W_{m e c h}}{c_{p}^{0}} .
$$

Now, from the mechanical energy balance (67), we can eliminate $W_{\text {mech }}$ in

favour of the overall compressible work of expansion/contraction $B$ and total viscous dissipation $D(K E)$, which yields:

$$
\int_{V} \rho \dot{\Theta}_{\mathrm{irr}}^{\mathrm{diff}} \mathrm{d} V=-\frac{B}{c_{p}^{0}}+\int_{V}\left(\frac{T-\theta}{T}\right) \frac{\rho \varepsilon_{K}}{c_{p}^{0}} \mathrm{~d} V \approx-\frac{B}{c_{p}^{0}},
$$

where the term involving viscous dissipation can be neglected owing to the fact that $(T-\theta) / T \ll 1$ is very small in the oceans (This approximation also assumes that $B$ is of the same order of magnitude as $D(K E)$, which seems confirmed by published results about the mechanical energy budget of 
numerical ocean models discussed further in the text). It also neglects the geothermal flux, which according to Graham and McDougall (2013) could potentially significantly alter the result and the estimation of the nonconservation of CT. Here, it is neglected on the grounds that OGCMs still do not systematically include it.. Eq. (70) is an important result, which states that the diffusive part of the nonconservative production of Conservative Temperature is a direct measure of the overall work of expansion/contraction, a result previously obtained by Tailleux (2012).

Although the precise magnitude of $B$ and of compressible effects in the oceans is still a matter of debate, e.g., Tailleux (2009a), let us note that in the context of ocean Boussinesq modelling, this term is classically approximated by substituting the pressure by the Boussinesq pressure $P \rightarrow-\rho_{0} g z$, the mass element by the Boussinesq mass element $\mathrm{d} m \rightarrow \rho_{0} \mathrm{~d} V$, using the Taylor series expansion $v \approx\left(1 / \rho_{0}\right)-\left(\rho-\rho_{0}\right) / \rho_{0}^{2}$ it follows that $P D v / D t \approx$ $\left(-\rho_{0} g z\right)(-D \rho / D t) / \rho_{0}^{2}$, which yields:

$$
\int_{V} P \frac{D v}{D t} \mathrm{~d} m \approx \int_{V} \rho_{0} g z \frac{1}{\rho_{0}^{2}} \frac{D \rho}{D t} \rho_{0} \mathrm{~d} V=\int_{V} g z \frac{D \rho}{D t} \mathrm{~d} V .
$$

In Boussinesq ocean models with a realistic nonlinear equation of state, density is nonconservative, and obeys an equation of the form:

$$
\frac{D \rho}{D t}=\nabla \cdot \mathbf{F}_{\rho}+\dot{\rho}_{i r r}
$$

where $\mathbf{F}_{\rho}$ it the diffusive flux of density due to the turbulent mixing of temperature and salinity, whereas $\dot{\rho}_{i r r}$ represents the effects due to the nonlinearities of the equation of state (Including compressibility effects, assumed to be small relative to the effects of cabelling and thermobaricity). This term is dominated by cabelling in the upper stratified ocean, but by thermobaricity 
in the weekly stratified abyssal ocean, as discussed by Oliver and Tailleux (2013). Inserting (72) into (71) allows the latter to be rewritten as:

$$
\int_{V} g z \frac{D \rho}{D t} \mathrm{~d} V \approx \underbrace{\int_{V} \rho_{0} K_{v} N^{2} \mathrm{~d} V}_{>0}+\underbrace{\int_{V} g z \dot{\rho}_{i r r} \mathrm{~d} V}_{<0} .
$$

The first term is positive and is associated with the classical result that mixing raises the centre of gravity of a fluid with a linear equation of state. The second term is in general dominated by cabelling and associated with contraction upon mixing, which plays a dominant role in the ocean energy budget, as perhaps first discussed by Gnanadesikan et al. (2005). In a steady-state, (71) can equivalently be rewritten in the following three equivalent forms:

$$
\int_{V} g z \frac{D \rho}{D t} \mathrm{~d} V=-\int_{V} \mathbf{u} \cdot \nabla_{h} P \mathrm{~d} V=-\int_{V} \rho g w \mathrm{~d} V
$$

which can all provide the basis for estimating $B$ using OGCM results, as reviewed in Tailleux (2013). The first estimation of $B$ for a realistic ocean model configuration is perhaps due to Toggweiler and Samuels (1998), based on the last expression in (74), who were the first to suggest that $B$ is negative in the ocean, rather than positive, in contrast to what was previously hypothesised by Oort et al (1994). Specifically, they find for the volume-averaged value of $B \approx 1.12 \times 10^{-6} \mathrm{erg} \mathrm{cm}^{-3} \mathrm{~s}^{-1}=1.12 \times 10^{-13} \mathrm{~J} \times 10^{6} \mathrm{~m}^{-3} \mathrm{~s}^{-1}=1.12 \times$ $10^{-7} \mathrm{Wm}^{-3}$. The ocean volume is about $V_{o c}=1.3 \times 10^{6} \mathrm{~km}^{3}=1.3 \times 10^{18} \mathrm{~m}^{3}$, resulting in a net energy conversion of $1.456 \times 10^{11} \mathrm{~W}=0.14 \mathrm{TW}$. In Gregory and Tailleux (2011), the value of $B$ in HadCM3 control climate is $-0.494 \mathrm{TW}$, based on the second expression in (74), while in the low resolution version FAMOUS it is $-0.060 \mathrm{TW}$. In $4 \times C O 2$ control climate, estimates of $B$ in both FAMOUS and HadCM3 are $O(0.12-0.13$ TW) (and negative), and hence similar to Toggweiler and Samuels (1998) estimates. 
409

\subsection{A priori estimates of nonconservative entropy production}

The second law of thermodynamics imposes the nonconservative production of entropy by molecular diffusive processes and viscous processes to be strictly positive. In a steady-state ocean, internal entropy production must be furthermore balanced by export of entropy by surface heat and freshwater fluxes. This can be expressed as:

$$
\int_{V} \rho \dot{\eta}_{i r r} \mathrm{~d} V \approx-\int_{S} \frac{Q_{\text {net }}}{T} \mathrm{~d} S=\frac{Q_{\text {out }}}{T_{\text {out }}}-\frac{Q_{\text {in }}}{T_{\text {in }}}
$$

$$
=\frac{Q_{\text {in }}+W_{\text {mech }}}{T_{\text {out }}}-\frac{Q_{\text {in }}}{T_{\text {in }}}=\frac{1}{T_{\text {out }}}\left[\left(\frac{T_{\text {in }}-T_{\text {out }}}{T_{\text {in }}}\right) Q_{\text {in }}+W_{\text {mech }}\right],
$$

where $1 / T_{\text {in }}$ is the weighted average of $1 / T$ restricted to regions where $Q_{\text {net }}>$ 0 , and $1 / T_{\text {out }}$ the weighted average of $1 / T$ over regions where $Q_{\text {net }}<0$. In the ocean, the mechanical power input $W_{\text {mech }}$ is strictly positive, while heating takes place on average at higher temperatures than cooling, as is expected from a heat engine (see related discussion by Tailleux (2010b)) so that $\left(T_{\text {in }}-T_{\text {out }}\right) Q_{\text {in }}>0$. As a result, the right-hand side of $(75)$ is also strictly positive, and therefore in agreement with the second law.

As for Conservative Temperature, it is useful to separate the diffusive and viscous contributions to the nonconservative entropy production, viz.,

$$
\int_{V} \rho \dot{\eta}_{\mathrm{irr}} \mathrm{d} V=\int_{V} \rho \dot{\eta}_{\mathrm{irr}}^{\mathrm{diff}} \mathrm{d} V+\int_{V} \frac{\rho \varepsilon_{K}}{T} \mathrm{~d} V=\int_{V} \rho \dot{\eta}_{\mathrm{irr}}^{\mathrm{diff}} \mathrm{d} V+\frac{D(K E)}{T_{\varepsilon}} .
$$

where the 'viscous' temperature $T_{\varepsilon}$ is defined so as to make the above equality exact, e.g., see Tailleux (2010b). By using the mechanical energy balance $W_{\text {mech }}+B=D(K E)$, we can eliminate $W_{\text {mech }}$ from Eq. (75) in favour of $B$ and $D(K E)$ to obtain the following expression for the diffusive part of the 
irreversible entropy production:

419

$$
\int_{V} \rho \dot{\eta}_{\text {irr }}^{\text {diff }} \mathrm{d} V=\left(\frac{T_{\text {in }}-T_{\text {out }}}{T_{\text {in }} T_{\text {out }}}\right) Q_{\text {in }}-\frac{B}{T_{\text {out }}}+\left(\frac{1}{T_{\text {out }}}-\frac{1}{T_{\varepsilon}}\right) D(K E)
$$

$$
\approx \frac{1}{T_{\text {out }}}\left[\left(\frac{T_{\text {in }}-T_{\text {out }}}{T_{\text {in }}}\right) Q_{\text {in }}+c_{p}^{0} \int_{V} \rho \dot{\Theta}_{\text {irr }}^{\text {diff }} \mathrm{d} V\right] .
$$

To arrive at (77), we neglected the term proportional to the overall viscous dissipation $D(K E)$ relative to the term proportional to $Q_{i n}$, while we replaced the compressible thermodynamic work term $B$ by its expression in terms of the nonconservative production of potential enthalpy. Eq. (77) is a useful result, which helps understand the link between the nonconservative production of potential enthalpy and entropy. One important remark of McDougall (2003) and Graham and McDougall (2013) is that entropy is considerably more nonconservative than potential enthalpy; in order for this to be true, Eq. (77) requires that the following inequality be satisfied:

$$
T_{\text {out }} \int_{V} \rho \dot{\eta}_{\mathrm{irr}}^{\mathrm{diff}} \approx\left(\frac{T_{\text {in }}-T_{\text {out }}}{T_{\text {in }}}\right) Q_{\text {in }} \gg\left|c_{p}^{0} \int_{V} \rho \dot{\Theta}_{\mathrm{irr}}^{\mathrm{diff}} \mathrm{d} V\right| .
$$

We can use published estimates of entropy production by surface heat fluxes to convince oneself that this inequality must indeed be satisfied in the ocean. For instance, Pascale et al (2011) estimate that the average entropy production is $O\left(1 \mathrm{~mW} \cdot \mathrm{K}^{-1} \cdot \mathrm{m}^{-2}\right)$ and hence that the total entropy production is of the order of $3.10^{11} \mathrm{~W} \cdot \mathrm{K}^{-1}$, using the result that the total area of the ocean is approximately $3.10^{14} \mathrm{~m}^{2}$. Using $T_{\text {out }} \approx 285 \mathrm{~K}$, the consequence is that $\left(T_{\text {in }}-T_{\text {out }}\right) Q_{\text {in }} / T_{\text {out }}$ must be of the order $85.5 \times 10^{12} \mathrm{~W}=85.5 \mathrm{TW}$. This value is in between one and two orders of magnitude larger than estimates for $W_{\text {mech }}$, which confirms that it is therefore expected to be much larger than estimates for $B$ and hence of potential enthalpy nonconservative production. 
This is consistent with McDougall (2003) conclusion that nonconservative production of potential enthalpy is about 2 orders of magnitude smaller than the nonconservative production of entropy scaled by $T_{\text {out }}$. The above shows that such a conclusion can be arrived at using much simpler arguments based on global budgets. A caveat should be mentioned, however, which is due to the fact that in contrast to entropy or Conservative Temperature, whose nonconservation is usually sign-definite, the nonconservation of $\theta$ can be of either sign. Since our approach focuses on global budgets, it will therefore underestimates the nonconservation of of potential temperature as compared to Graham and McDougall (2013), which focuses on the root-mean square of locally estimated nonconservation terms.

Note that our approach here is very different from Yan et al. (2004), who estimate the irreversible entropy production due to radiative heat fluxes to be at least two orders of magnitude greater than the one discussed in Pascale et al (2011). The difference arises because Yan et al. (2004) considers that upon thermalisation with the ocean, the entropy of radiation increases from its very low value $F_{\text {sw }} / T_{\text {sun }}$ upon leaving the sun to the very high value $F_{\text {sw }} / T_{\text {ocean }}$ upon thermalisation with matter (i.e, seawater here), where $T_{\text {sun }}$ and $T_{\text {ocean }}$ are the temperatures of the sun and ocean surface respectively. As a result, their entropy budget is dominated by terms like $F_{\text {sw }}\left(1 / T_{\text {sun }}-1 / T_{\text {ocean }}\right)$, but arguably, this irreversible production term is better viewed as part of the sun+photons+earth system, rather than as part of the ocean. See also Pelkowski (2014) for a recent discussion of the entropy of radiation. The viewpoint taken here is that upon thermalisation, photons lose their identity, and just contribute to increase the energy levels of the 
468

matter with which they interact. This interaction is most easily treated as regular 'heating', and does not require the knowledge of the initial entropy of the photons at the time they were emitted by the sun.

\subsection{A priori estimates of nonconservative production of $\theta$}

We now apply the above ideas to the problem of deriving a priori estimates for the nonconservative production of potential temperature, which leads us to rewrite (65) as follows:

$$
\int_{V} \rho \dot{\theta}_{i r r} \mathrm{~d} V=-\int_{S} \frac{Q_{n e t}}{c_{p}^{R}} \mathrm{~d} S=\frac{Q_{\text {out }}}{c_{p, \text { out }}^{R}}-\frac{Q_{\text {in }}}{c_{p, \text { in }}^{R}}
$$

$$
=\frac{Q_{\text {in }}+W_{\text {mech }}}{c_{p, \text { out }}^{R}}-\frac{Q_{\text {in }}}{c_{p, \text { in }}^{R}}=\frac{1}{c_{p, \text { out }}^{R}}\left[\left(\frac{c_{p, \text { in }}^{R}-c_{p, \text { out }}^{R}}{c_{p, \text { in }}^{R}}\right) Q_{\text {in }}+W_{\text {mech }}\right],
$$

where $c_{p, \text { in }}^{R}$ and $c_{p, \text { out }}^{R}$ are the reciprocal of weighted means of $1 / c_{p}^{R}$ averaged over the regions of net heating and cooling respectively, and defined so as to make the above decomposition exact. Eq. (79) shows that the nature of the potential temperature nonconservation is in many ways similar to that for entropy, given that (79) is essentially identical in structure to (75) with $c_{p}^{R}$ in the former replacing $T$ in the latter. As previously, we separate the nonconservative production of $\theta$ into a diffusive and viscous part, so that (79) becomes:

$$
\int_{V} \rho \dot{\theta}_{\mathrm{irr}}^{\mathrm{diff}} \mathrm{d} V+\frac{D(K E)}{c_{p, \varepsilon}^{R}}=\left(\frac{c_{p, \text { in }}^{R}-c_{p, \text { out }}^{R}}{c_{p, \text { in }}^{R} c_{p, \text { out }}^{R}}\right) Q_{\text {in }}+\frac{W_{\text {mech }}}{c_{p, \text { out }}^{R}},
$$

where we defined the quantity $c_{p, \varepsilon}^{R}$ via the relation:

$$
\frac{\int_{V} \rho \varepsilon_{K}}{c_{p, \varepsilon}^{R}}=\int_{V} \frac{\theta}{T c_{p}^{R}} \rho \varepsilon_{K} \mathrm{~d} V .
$$


As a result, we obtain the following expression for the diffusive part of $\dot{\theta}_{i r r}$ :

$$
\int_{V} \rho \dot{\theta}_{\mathrm{irr}}^{\mathrm{diff}} \mathrm{d} V=\left(\frac{c_{p, \text { out }}^{R}-c_{p, \text { in }}^{R}}{c_{p, \text { in }}^{R} c_{p, \text { out }}^{R}}\right) Q_{\text {in }}-\frac{B}{c_{p, \text { out }}^{R}}+\left(\frac{1}{c_{p, \text { out }}^{R}}-\frac{1}{c_{p, \varepsilon}^{R}}\right) \int_{V} \rho \varepsilon_{K}
$$

477

$$
\approx \frac{1}{c_{p, \text { out }}^{R}}\left[c_{p}^{0} \int_{V} \rho \dot{\Theta}_{\mathrm{irr}}^{\mathrm{diff}}+\left(\frac{c_{p, \text { in }}^{R}-c_{p, \text { out }}^{R}}{c_{p, \text { in }}^{R}}\right) Q_{\text {in }}\right]
$$

where the approximation was obtained by neglecting the term proportional to viscous dissipation relative to the term proportional to $Q_{i n}$. Eq. (81) is a key result of this paper, for it provides an explicit expression for the difference between the nonconservative production of potential and Conservative Temperature, which appears to be controlled by the net heating $Q_{i n}$, as well as by the spatial variations of the heat capacity at the surface. One of McDougall (2003) key conclusions is that the overall nonconservative production of Conservative Temperature should be about two orders of magnitude smaller than the non-conservation of potential temperature. According to (81), this can be the case only if the following constraint is satisfied:

$$
\left|c_{p, \text { out }}^{R} \int_{V} \rho \dot{\theta}_{\text {irr }}^{\text {diff }} \mathrm{d} V\right| \approx\left|\left(\frac{c_{p, \text { in }}^{R}-c_{p, \text { out }}^{R}}{c_{p, \text { in }}^{R}}\right) Q_{\text {in }}\right| \gg\left|c_{p}^{0} \int_{V} \rho \dot{\Theta}_{\text {irr }}^{\text {diff }}\right| .
$$

Physically, this constraint requires that the weighted averaged heat capacity over heating regions be significantly different than the weighted averaged heat capacity over the cooling regions. Why this should be the case in the ocean is unclear, and the nature of the spatial variations in $c_{p}^{R}$ giving rise to such a big difference is addressed empirically in the next section using observations. Eq. (81) shows that the exact difference between the net nonconservative production of potential and Conservative Temperature depends on the particular circumstances of the system studied, and is not entirely 
an intrinsic property of the conservative versus potential temperature, in the sense that for a fluid with nearly constant heat capacity, potential temperature would be nearly as conservative as Conservative Temperature. In fact, the net nonconservative production of $\theta$ could be even less than for $\Theta$ if the the surface ocean properties were such as to make the two terms within the square brackets in (81) cancel out. Whether such a configuration could occur as a result of changes in the state of our climate is left as an intriguing open question, whose answer could perhaps give us important clues about the functioning of the ocean/atmosphere coupling.

\section{Observational constraints on nonconservative effects}

\subsection{Data sources}

In this section, we use freely available climatological datasets to estimate the two surface integrals

$$
\begin{aligned}
& -\int_{S} \frac{Q_{n e t}}{T} \mathrm{~d} A, \\
& -\int_{S} \frac{Q_{n e t}}{c_{p}^{R}} \mathrm{~d} A,
\end{aligned}
$$

which we showed above to dominate the diffusive part of the nonconservative production of entropy and potential temperature. The estimation of these two integrals requires the knowledge of surface values of temperature and salinity (to estimate $c_{p}^{R}$ ), as well as estimates of the net heat flux into the oceans. The computations discussed in the following sections were obtained by using the annual mean surface temperature and salinity from the World Ocean Database 2013, as well as the balanced NOCS net heat flux product discussed in Grist and Josey (2003). 


\subsection{Observational constraints on irreversible entropy production}

As mentioned by Grist and Josey (2003), most available heat flux products are unbalanced. Even the balanced NOCS heat product version 1.1a depicted in Fig. 1, which is obtained through an inverse method is still unbalanced by $-5 \mathrm{~W} \cdot \mathrm{m}^{-2}$, which amounts a net cooling of about $-1.510^{15} \mathrm{~W}=$ $-1.5 \mathrm{PW}$. Were the oceans to be in a true steady-state and to satisfy the global energy balance $Q_{\text {cooling }}=Q_{i n}+W_{\text {mech }}$, the net cooling should exceed the net heating so as to cancel out the Joule heating arising from the viscous dissipation of the power input due to the mechanical sources of energy. The net heating is so much larger than the power input by mechanical sources of energy $\left(Q_{\text {in }}=O(2 \mathrm{PW})\right.$ versus $\left.W_{\text {mech }}=O(1-10 \mathrm{TW})\right)$ that it is very difficult to ascertain that the observed imbalance in available heat flux products occur because of the need to balance $W_{\text {mech }}$, or as the result of the large uncertainties plaguing the evaluation of the various terms entering the heat budget. The NOCS heat flux product comes in two different versions, one that is unbalanced, and another one that was balanced using an inverse method.

To assess the role of imbalance in heat flux products, we write the net heat flux $Q_{n e t}=\bar{Q}+\tilde{Q}$ as the sum of a constant spatially uniform component $\bar{Q}$ plus a component $\tilde{Q}$ that integrates to zero. The entropy integral (83) thus becomes:

$$
-\int_{S} \frac{Q_{n e t}}{T} \mathrm{~d} S=-\bar{Q} \int_{S} \frac{d S}{T}-\int_{S} \frac{\tilde{Q}}{T} \mathrm{~d} S=-\frac{A_{o c} \bar{Q}}{\bar{T}}-\frac{\tilde{Q}_{\text {in }}}{T_{\text {in }}}+\frac{\tilde{Q}_{\text {out }}}{T_{\text {out }}}
$$

where $\bar{T}$ is the geometric average of the surface temperature, while $\tilde{Q}_{i n}$ and $\tilde{Q}_{\text {out }}$ are now defined to balance exactly, i.e., to satisfy $\tilde{Q}_{i n}=\tilde{Q}_{\text {out }}$, with $A_{o c}$ 
the surface area of the oceans. Here, the imbalance in the NOCS 1.1a heat flux product is of the order $\bar{Q} \approx 5 \mathrm{~W} \cdot \mathrm{m}^{-2}$, so that

$$
-\frac{A_{o c} \bar{Q}}{\bar{T}} \approx \frac{3.10^{14} \mathrm{~m}^{2} \times 5 \mathrm{~W} \cdot \mathrm{m}^{-2}}{291} \approx 5.10^{12} \mathrm{~W} \cdot \mathrm{K}^{-1} \text {. }
$$

In the present case, the imbalance in the heat flux product is so large that if retained, it would be the term dominating the entropy budget. In reality, we expect the contribution from $\tilde{Q}_{\text {in }}$ and $\tilde{Q}_{\text {out }}$ to dominate.

We can try to anticipate the results of observational computation by using scaling argument, based on using the following typical values $\tilde{Q}_{i n}=2.10^{15} \mathrm{~W}$, $T_{\text {in }}-T_{\text {out }}=15^{\circ} \mathrm{C}, T_{\text {in }} T_{\text {out }} \approx 300^{2} K^{2}$, which gives us

$$
\frac{T_{\text {in }}-T_{\text {out }}}{T_{\text {in }} T_{\text {out }}} \tilde{Q}_{\text {in }} \approx \frac{15 \times 2.10^{15}}{300^{2}}=0.33 \times 10^{12} \mathrm{~W} . \mathrm{K}^{-1}
$$

This value is equal to $1.1 \mathrm{~mW} \cdot \mathrm{m}^{-2} \cdot \mathrm{K}^{-1}$ when divided by the area of the ocean, which is comparable to that estimated in the UK Hadley Centre coupled climate model HadCM3 by Pascale et al (2011). Physically, it is also useful to decompose the total temperature $T=\bar{T}+T^{\prime}$ into a mean and perturbation part, in order to approximate entropy production as

$$
-\int_{S} \frac{\tilde{Q}}{T} \mathrm{~d} S \approx \frac{1}{\bar{T}^{2}} \int_{S} \tilde{Q} T^{\prime} \mathrm{d} S
$$

which shows that the diffusive part of entropy production is primarily controlled by the degree of correlation between the surface heat flux and the surface temperature anomalies. Fig. 2 shows the spatial map of the leading order term $-\tilde{Q} / \bar{T}$ and the second order term $\tilde{Q} T^{\prime} / \bar{T}^{2}$ in entropy production. The leading order term is a rescaled version of the net heat flux depicted in Fig. 1. Numerical estimates yield $0.3 \times 10^{12} \mathrm{~W} . \mathrm{K}^{-1}$ for the total entropy production, which is nearly identical to the scaling argument above. Multiplying 
this term by $\bar{T}=291 \mathrm{~K}$ yields $87 \mathrm{TW}$, which is the number to be compared with the non conservation of potential enthalpy, following McDougall (2003) and Graham and McDougall (2013).

\subsection{Observational constraints on irreversible production of $\theta$}

Leaving out the unbalanced part of the heat flux, (84) becomes:

$$
\int_{V} \rho \dot{\theta}_{\text {irr }}^{\text {diff }} \mathrm{d} V \approx-\int_{S} \frac{\tilde{Q}}{c_{p}^{R}} \mathrm{~d} S=\frac{\tilde{Q}_{\text {out }}}{c_{p, \text { out }}^{R}}-\frac{\tilde{Q}_{\text {in }}}{c_{p, \text { in }}^{R}}=\left(\frac{1}{c_{p, \text { out }}^{R}}-\frac{1}{c_{p, \text { in }}^{R}}\right) \tilde{Q}_{\text {in }}
$$

so that fundamentally, the non conservation of potential temperature arises principally from the spatial variations of $c_{p}^{R}$ due to the spatial variations of surface temperature and salinity. In this paper, we use the climatological annual mean values of temperature and salinity depicted in Fig. 3. The implied variations in $c_{p}$ are depicted in the bottom panel of Fig. 4, while the top panel illustrates the dependence of $c_{p}$ on temperature and salinity. The spatial distribution of the leading order contribution $-\tilde{Q} / c_{p}^{R}\left(\bar{S}, \bar{\theta}, P_{0}\right)$ (where $c_{p}^{R}\left(\bar{S}, \bar{\theta}, P_{0}\right)$ is spatially uniform) is illustrated in the top left panel of Fig. 5, while the the second order contribution (due to the departure of $c_{p}^{R}$ from its mean), is depicted in the top right panel. As in the case of entropy production, the leading order term is merely a rescaled version of the net heat flux illustrated in Fig. 1. Only the second order contribution, however, contributes to the surface integral, found to be $\bar{c}_{p} \int_{V} \rho \dot{\theta}_{i r r}^{\text {diff }} \approx 4.6 \mathrm{TW}$. In order to understand the physical origin of this number, it is useful to examine the relative contribution of the temperature and salinity dependence of $c_{p}^{R}$ on the result. To that end, we can use a Taylor series expansion to write:

$$
\frac{1}{c_{p}^{R}} \approx \frac{1}{\bar{c}_{p}}-\frac{1}{\bar{c}_{p}^{2}} \frac{\partial c_{p}}{\partial T}\left(\bar{T}, \bar{S}, P_{0}\right)(T-\bar{T})-\frac{1}{\bar{c}_{p}^{2}} \frac{\partial c_{p}}{\partial S}\left(\bar{T}, \bar{S}, P_{0}\right)(S-\bar{S})
$$


where $\bar{c}_{p}=c_{p}\left(\bar{T}, \bar{S}, P_{0}\right)$, which in turns yields:

$$
-\int_{S} \frac{\tilde{Q}}{c_{p}^{R}} \mathrm{~d} S \approx \frac{1}{\bar{c}_{p}^{2}} \frac{\partial \bar{c}_{p}}{\partial T} \int_{S} \tilde{Q} T^{\prime} \mathrm{d} S+\frac{1}{\bar{c}_{p}^{2}} \frac{\partial \bar{c}_{p}}{\partial S} \int_{S} \tilde{Q} S^{\prime} \mathrm{d} S .
$$

Fig. 6 shows that $c_{p}$ is more sensitive to salinity than to temperature. However, the relative contribution of temperature and salinity anomalies in (90) is not a priori obvious, because even though the dependence of $c_{p}$ on temperature is much smaller than on salinity, salinity anomalies do not correlate strongly with heat flux anomalies, in contrast to temperature anomalies. The numerical evaluation of the two terms in (90) supports this, with the temperature and salinity terms being approximately $3.6 \mathrm{TW}$ and $1 \mathrm{TW}$ respectively, thus showing that it is actually the $c_{p}$ dependence on temperature that ultimately dominates, which differs from McDougall (2003).

\section{Summary and implications for ocean modelling}

The results of this paper make it clear that the non conservation of potential temperature and that of Conservative Temperature are fundamentally different, since the non conservation of the former primarily reflects its production/destruction by surface fluxes, whereas the non conservation of the latter is primarily a measure of the thermodynamic work of expansion/contraction (in a steady-state). A a result, the only way to preserve the balance between surface and interior production/destruction established for the non-averaged Navier-Stokes equations would require to replace the evolution equation and boundary conditions for potential temperature used 


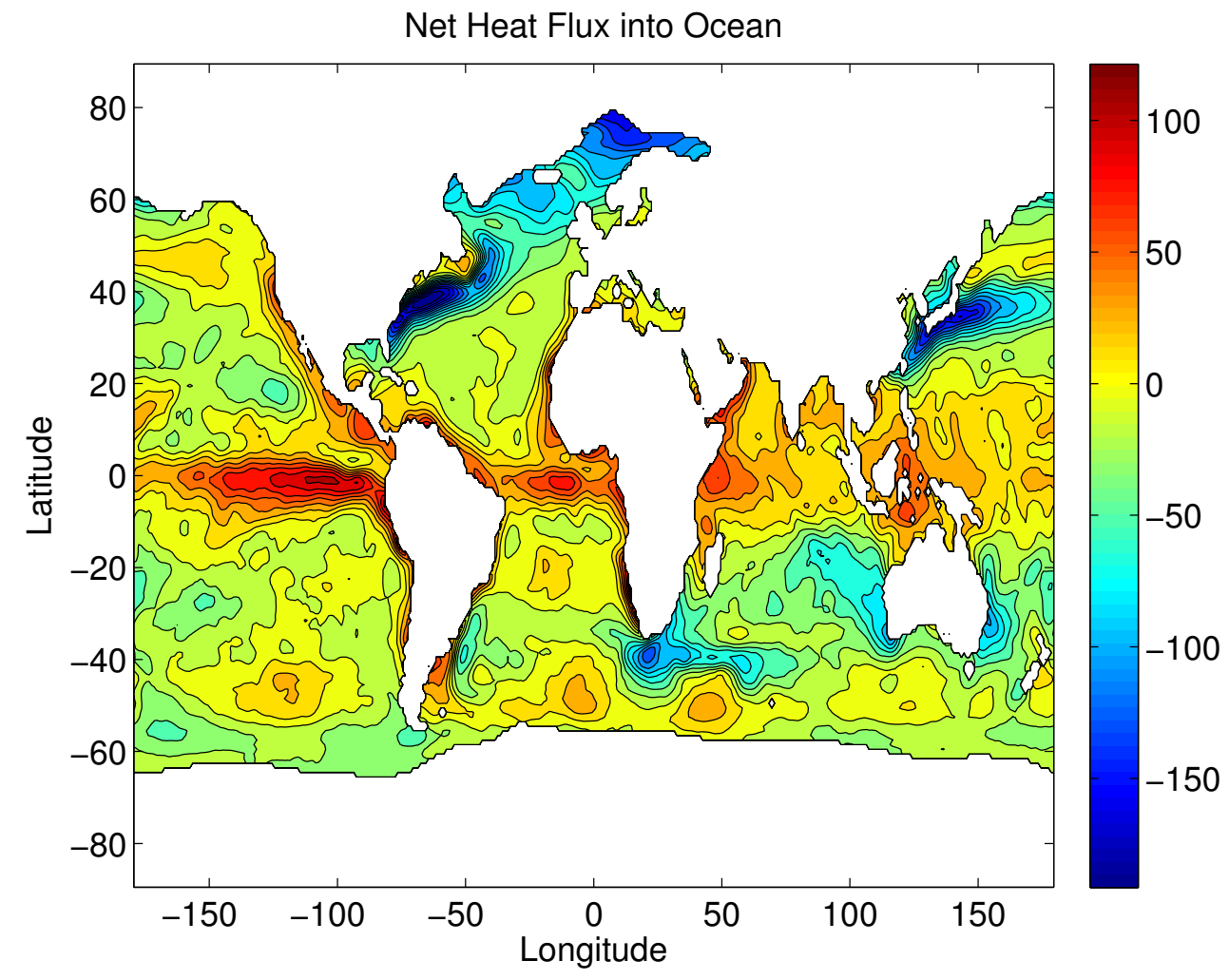

Figure 1: Net heat flux into the ocean in $\mathrm{W} \cdot \mathrm{m}^{-2}$ from the balanced NOCS 1.1a heat product.

600

in current OGCM formulations by the following ones

$$
\frac{D \theta}{D t}=-\nabla \cdot \mathbf{F}_{\theta} \quad \rightarrow \quad \frac{D \theta}{D t}=-\frac{1}{c_{p}^{R}} \nabla \cdot\left(c_{p}^{R} \mathbf{F}_{\theta}\right)+N . C .
$$

$$
K \frac{\partial \theta}{\partial z}(z=0)=\frac{Q_{\text {sens }}}{\rho_{0} c_{p 0}} \quad \rightarrow \quad K \frac{\partial \theta}{\partial z}(z=0)=\frac{Q_{\text {sens }}}{\rho_{0} c_{p}^{R}},
$$

where N.C. denotes additional nonconservative effects discussed below, with corresponding changes required for the radiative and latent heat fluxes, and $K$ a vertical turbulent eddy diffusivity.

In order to fully specify the form of the potential temperature equation 
(91), one needs a way to express its nonconservative part in terms of the turbulent fluxes of heat and salt. Tailleux (2010a) and Graham and McDougall (2013) both show that the form of the nonconservative part follows from assuming a certain quantity (in addition to salinity) to be conservative, but they disagree on which one. Specifically, Tailleux (2010a) assumes it to be total energy, as for the non-averaged Navier-Stokes equations, whereas Graham and McDougall (2013) use a pseudo-conservative quantity - namely a locally-referenced form of potential enthalpy — which they take to vary from one grid-point to the next, so that although it is treated as conservative for the purpose of estimating the non conservation of $\theta$ and CT, it is actually nonconservative from a strict mathematical viewpoint (hence referred to here as pseudo-conservative). Despite being based on different approaches, the expressions obtained by Graham and McDougall (2013) and Tailleux (2010a) are quite similar, the main difference being the former lacking the terms proportional to the pressure gradient of the latter.

For lack of definite understanding about how to handle the non conservation of potential temperature, IOC et al. (2010) recommended that OGCMs should adopt Conservative Temperature and Absolute Salinity as their new prognostic variables, on the grounds that the non conservation of such quantities is sufficiently small to justify treating them as exactly conservative, and hence governed by

$$
\frac{D \Theta}{D t}=-\nabla \cdot \mathbf{F}_{\Theta}, \quad \frac{D S_{A}}{D t}=-\nabla \cdot \mathbf{F}_{S}
$$

(with additional terms required to handle radiation and latent heat release in the CT equation). Turbulent fluxes are expressed in terms of a diffusivity 
tensor $\mathbf{K}$, including diapycnal, mixing and eddy-induced transport, as follows

$$
\mathbf{F}_{S}=-\mathbf{K} \nabla S_{A}, \quad \mathbf{F}_{\Theta}=-\mathbf{K} \nabla \Theta .
$$

It is important to recognise, however, that switching to $\left(\Theta, S_{A}\right)$ as prognostic variables necessitates the specification of explicit functional relationships $T=T\left(\Theta, S_{A}, P\right)$ and $\theta=\theta\left(\Theta, S_{A}, P\right)$ allowing one to invert $T$ and $\theta$ from the knowledge of $\Theta, S_{A}$ and $P$. Such inverse relations are available in the form of Matlab subroutines as part of the Gibbs Sea Water (GSW) Library (available at www.teos-10.org) called gsw_t_from_CT.m and gsw_pt_from_CT.m for instance. Since such inverse relations are known from basic thermodynamic principles, they can be differentiated in order to obtain $\mathrm{d} \theta$ as a function of $\mathrm{d} \Theta$ and $\mathrm{d} S_{A}$, which are none other than the passage relations (14) and (15) derived previously. Thus, Eq. (15) yields the following equation for $D \theta / D t$,

$$
\frac{D \theta}{D t}=\frac{c_{p}^{0}}{c_{p}^{R}} \frac{D \Theta}{D t}-\frac{1}{c_{p}^{R}}\left(\mu_{R}-\theta \frac{\partial \mu_{R}}{\partial \theta}\right) \frac{D S_{A}}{D t},
$$

while (14) yields the following expression for $\nabla \Theta$

$$
\nabla \Theta=\frac{1}{c_{p}^{0}}\left(\mu_{R}-\theta \frac{\partial \mu_{R}}{\partial \theta}\right) \nabla S_{A}+\frac{c_{p}^{R}}{c_{p}^{0}} \nabla \theta .
$$

It is now straightforward to combine (93), (94), (95) and (95) to deduce what the evolution equation for $\theta$ and turbulent flux $\mathbf{F}_{\theta}$ should be to be equivalent to the proposed IOC et al. (2010) $\left(\Theta, S_{A}\right)$ formulation, viz.,

$$
\frac{D \theta}{D t}=-\frac{1}{c_{p}^{R}} \nabla \cdot\left(c_{p}^{R} \mathbf{F}_{\theta}\right)-\frac{1}{c_{p}^{R}} \mathbf{F}_{S} \cdot \nabla\left(\mu_{R}-\theta \frac{\partial \mu_{R}}{\partial \theta}\right),
$$

$$
\mathbf{F}_{\theta}=-\mathbf{K} \nabla \theta \text {. }
$$

As to the surface boundary condition for $\Theta,(14)$ shows that it should be

$$
K \frac{\partial \Theta}{\partial z}(z=0)=\frac{Q_{s e n s}}{\rho_{0} c_{p}^{0}}+\frac{1}{c_{p}^{0}}\left(\mu_{R}-\theta \frac{\partial \mu_{R}}{\partial \theta}\right) K \frac{\partial S_{A}}{\partial z}(z=0),
$$


644

$$
=\frac{Q_{\text {sens }}}{\rho_{0} c_{p}^{0}}+\frac{1}{c_{p}^{0}}\left(\mu_{R}-\theta \frac{\partial \mu_{R}}{\partial \theta}\right) \frac{\rho_{f} S(E-P)}{\rho}
$$

645

654

655

and hence that it should contain a term proportional to the salinity boundary condition, which is consistent with the fact that it is the reduced heat flux $\mathbf{F}_{q}=F_{h}-(\partial h / \partial S) \mathbf{F}_{S}$, rather than $\mathbf{F}_{h}$, which is linked to the sensible heat flux. This is an important point that is mentioned neither in McDougall (2003) nor in IOC et al. (2010), but which would require an additional modification to existing codes when switching to a $\left(\Theta, S_{A}\right)$ formulation. In order to assess the relative merits of the $(\theta, S)$ versus $\left(\Theta, S_{A}\right)$ formulations, we find it useful to separate the conservative and nonconservative part of the right-hand side of (97) as follows

$$
\frac{D \theta}{D t}=-\nabla \cdot \mathbf{F}_{\theta}+\dot{\theta}_{i r r}
$$

The expression for the nonconservative term $\dot{\theta}_{i r r}$ can be further clarified by expanding the gradients of the different functions of $\theta$ and $S_{A}$, leading to

$$
\dot{\theta}_{i r r}=A\left(\theta, S_{A}\right) \nabla \theta^{T} \cdot(\mathbf{K} \nabla \theta)+B\left(\theta, S_{A}\right) \nabla S_{A}^{T} \cdot\left(\mathbf{K} \nabla S_{A}\right)
$$

$$
+C\left(\theta, S_{A}\right)\left[\nabla S_{A}^{T} \cdot(\mathbf{K} \nabla \theta)+\nabla \theta^{T} \cdot\left(\mathbf{K} \nabla S_{A}\right)\right]
$$

where $A, B$, and $C$ are all functions of $\theta$ and $S$ alone given by

$$
A\left(\theta, S_{A}\right)=\frac{1}{c_{p}^{R}} \frac{\partial c_{p}^{R}}{\partial \theta}, \quad B\left(\theta, S_{A}\right)=\frac{1}{c_{p}^{R}}\left(\frac{\partial \mu_{R}}{\partial S_{A}}-\theta \frac{\partial^{2} \mu_{R}}{\partial S_{A} \partial \theta}\right),
$$

$$
C\left(\theta, S_{A}\right)=\frac{1}{c_{p}^{R}} \frac{\partial c_{p}^{R}}{\partial S_{A}}=-\frac{\theta}{c_{p}^{R}} \frac{\partial^{2} \mu_{R}}{\partial \theta^{2}} .
$$

Given that the modifications to the potential temperature equation detailed above should be strictly equivalent to using the $\left(\Theta, S_{A}\right)$ formulation proposed 
by IOC et al. (2010), the actual benefits of switching to Conservative Temperature are no longer obvious. From a computational viewpoint, adding the nonconservative term (101) and modifying the current boundary condition as per (92) would be straightforward. Moreover, since OGCMs estimate $\nabla \theta$, $\nabla S_{A}, \mathbf{K} \nabla \theta$ and $\mathbf{K} \nabla S_{A}$ as part of computing the heat and salt fluxes, diagnosing $\dot{\theta}_{i r r}$ would come at little additional cost, as to do so would only require additional routines for the 4 functions of $\theta$ and $S_{A}$ alone, namely $c_{p}^{R}, A, B$, and $C$. As regards to estimating the poleward heat transport, it could easily be diagnosed in terms of CT as recommended by McDougall (2003), since $\Theta$ can be diagnosed from $\left(\theta, S_{A}\right)$ (using the routine gsw_CT_from_pt.m from the GSW Library for instance). Since a $\left(\theta, S_{A}\right)$ formulation can be constructed that is strictly equivalent to the $\left(\Theta, S_{A}\right)$ formulation proposed by IOC et al. (2010), it follows that the decision to switch to CT should be motivated on a careful evaluation of the computational and physical advantages of each formulation. Note, however, that even though the $\left(\theta, S_{A}\right)$ and $\left(\Theta, S_{A}\right)$ formulations discussed in this section are constructed to be strictly equivalent mathematically, it would be of interest to test whether this is also the case at the discretised level, as differences in results could shed light on whether the budget of derived nonconservative quantities such as buoyancy or entropy can be expected to be accurately represented in numerical ocean models.

\section{Acknowledgements}

Numerous discussions with Trevor McDougall over the past few years greatly helped in defining and clarifying the issues addressed in this paper, and contributed to point the way to the results of the last section. Con- 
structive criticism by three anonymous referees greatly helped clarify and improve the paper. This project was supported by the NERC funded INSPECT project NE/K016083/1.

Bacon, S. and N. Fofonoff (1996)Oceanic heat flux calculation. J. Atmos. Oceanic Technol., 24, 1785-1799

Beron-Vera, F.J., Ochoa, J., and Ripa, P. (1999): note on boundary conditions for salt and freshwater balances. Ocean Modelling, 1, 111-118

Brankart, J.-M. (2013): Impact of uncertainties in the horizontal density gradient upon low resolution global ocean modelling. Ocean Modelling, 66, 64-76

de Groot, S.R. and Mazur, P. (1962)Non-equilibrium thermodynamics. NorthHolland publishing company, Amsterdam.

Fofonoff, N. (1962)Physical properties of sea-water. In M.N. Hill (Ed.), The Sea (pp 3-30).New York: J. Wiley and Sons.

Gnanadesikan, A., R. D. Slater, P. S. Swathi, and G. K. Vallis. (2005) The energetics of ocean heat transport. J. Climate, 18, 2604-2616.

Graham, F. and McDougall, T. J. (2013) Quantifying the Nonconservative Production of Conservative Temperature, Potential Temperature, and Entropy. J. Phys. Oceanography, bf 43, 838-862.

Gregory, J. M. and R. Tailleux (2011)Kinetic energy analysis of the response of the Atlantic meridional overturning circulation to $\mathrm{CO}_{2}$-forced climate change. Clim. Dyn., 37, 893-914. 
Griffies, S.M., et al. (2009)Coordinated Ocean-ice Reference Experiments (COREs) Ocean Modelling, 26, 1-46

Grist, J.P. and S.A. Josey (2003)Inverse analysis adjustment of the SOC airsea flux climatology using ocean heat transport constraints. J. Climate, 16, 3274-3295

IOC, SCOR and IAPSO (2010)The international thermodynamic equation of seawater -2010: Calculation and use of thermodynamic properties. Intergovernmental Oceanographic Commission, Manuals and Guides No. 56, UNESCO (English). 196pp. Available from http://www.TEOS-10.org

Landau, L.D. and E.M. Lifshitz (1987)Fluid Mechanics 2nd edition. Course of Theoretical Physics. Volume 6. Pergamon Press.

McDougall, T. J. (2003)Potential enthalpy: a conservative oceanic variable for evaluating heat content and heat fluxes. J. Phys. Oceanogr.,, 33, 945963

Oliver, K.I.C. and R. Tailleux (2013)Thermobaric control of gravitational potential energy by diapycnal mixing in the deep ocean. Geophys. Res. Lett., 40, 327-331.

Oort, A.H., L.A. Anderson and J.P. Peixoto (1994)Estimates of the energy cycle of the oceans. J. Geophys. Res., 99, 7665-7688.

Pascale, S., J.M. Gregory, M. Ambaum, and R. Tailleux (2011) Climate entropy budget of the HadCM3 atmosphere ocean general circulation model and of FAMOUS, its low-resolution version. Clim. Dyn., 36, 1189-1206 
Pawlowicz, R., T.J. McDougall, R. Feistel, and R. Tailleux (2012) Preface: An historical perspective on the development of the Thermodynamics equation of seawater - 2010. Ocean Science, 8, 161-174.

Pelkowski, J. (2014)On the Clausius-Duhem inequality and maximum entropy production in a simple radiating system. Entropy, 16, 2291-2308.

Tailleux, R. (2009a)On the energetics of stratified turbulent mixing, irreversible thermodynamics, Boussinesq models and the ocean heat engine controversy. J. Fluid Mech., 638, 339-382.

Tailleux, R. (2010a)Identifying and quantifying nonconservative energy production/destruction in hydrostatic Boussinesq primitive equation models. Ocean Modelling, 34, 125-136.

Tailleux, R. (2010b)Entropy versus APE production: on the buoyancy power input in the oceans energy cycle. Geophys. Res. Lett. 37, Article ID L22603.

Tailleux, R. (2012)Thermodynamics/dynamics coupling in weakly compressible turbulent stratified fluids. ISRN Thermodynamics, Article ID 609701, doi:10.5402/2012/609701

Tailleux, R. (2013)Available potential energy and exergy in turbulent stratified fluid. Ann. Rev. Fluid Mech. 45, 35-58.

Toggweiler, J. R. and B. Samuels (1998)On the ocean's large-scale circulation near the limit of no vertical mixing. J. Phys. Oceanogr., 28, 1832-1852.

Warren, B. A. (2006)The First Law of Thermodynamics in a salty ocean. Prog. Oceanogr., 70, 149-167. 
749 Yan, Y., Z. Gan and Y. Qi (2004)Entropy budget of the ocean system. Geophys. Res. Lett., 31, L14311. doi:10.1029/2004GL019921 

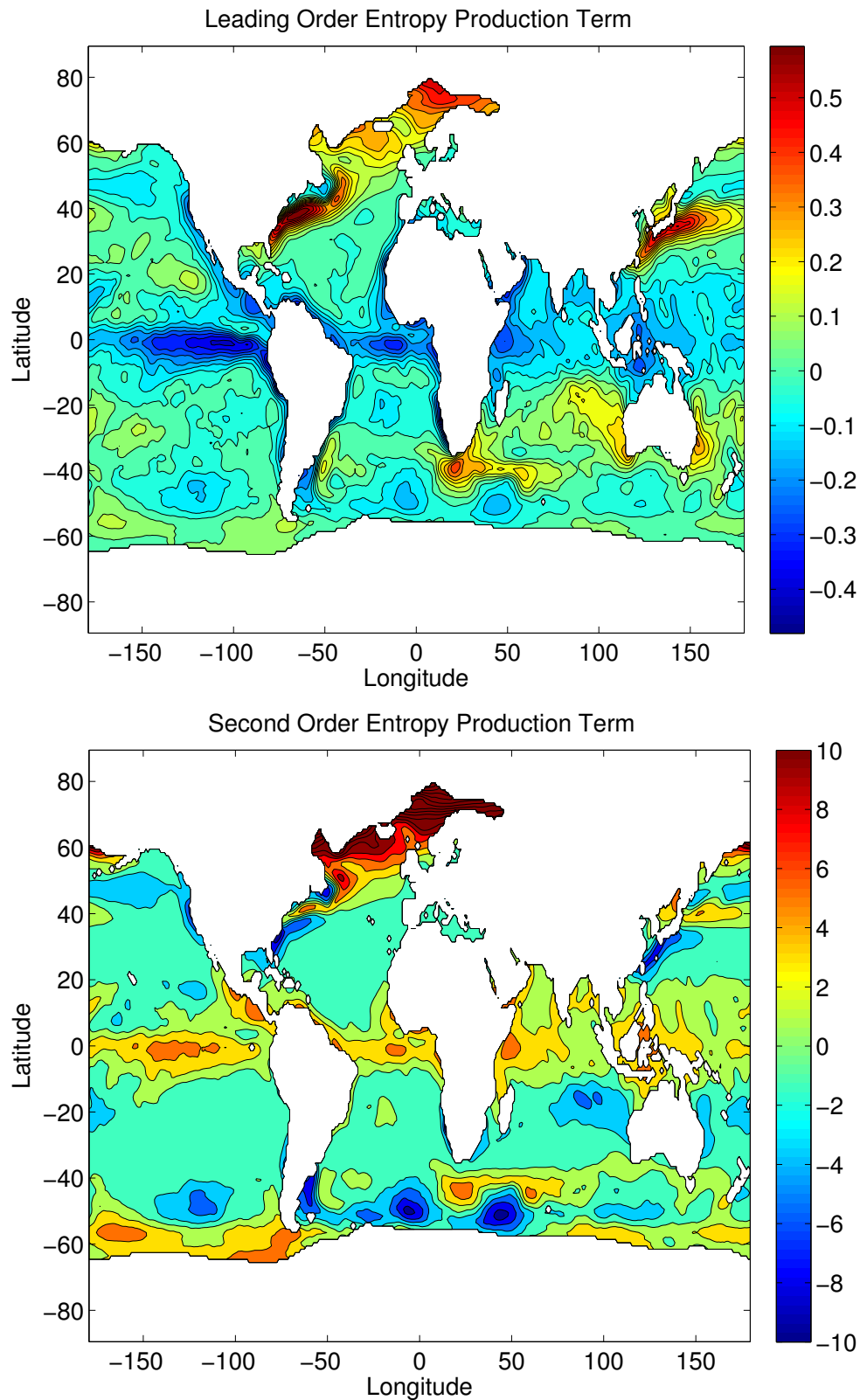

Figure 2: Leading order entropy production term $-\tilde{Q} / \bar{T}$ (top panel, in $\mathrm{W} \cdot \mathrm{m}^{-2} \cdot \mathrm{K}^{-1}$ ) and second order entropy production term $-\tilde{Q}(1 / T-1 / \bar{T})$ (bottom panel, in $\mathrm{mW} \cdot \mathrm{m}^{-2} \cdot \mathrm{K}^{-1}$ ) 

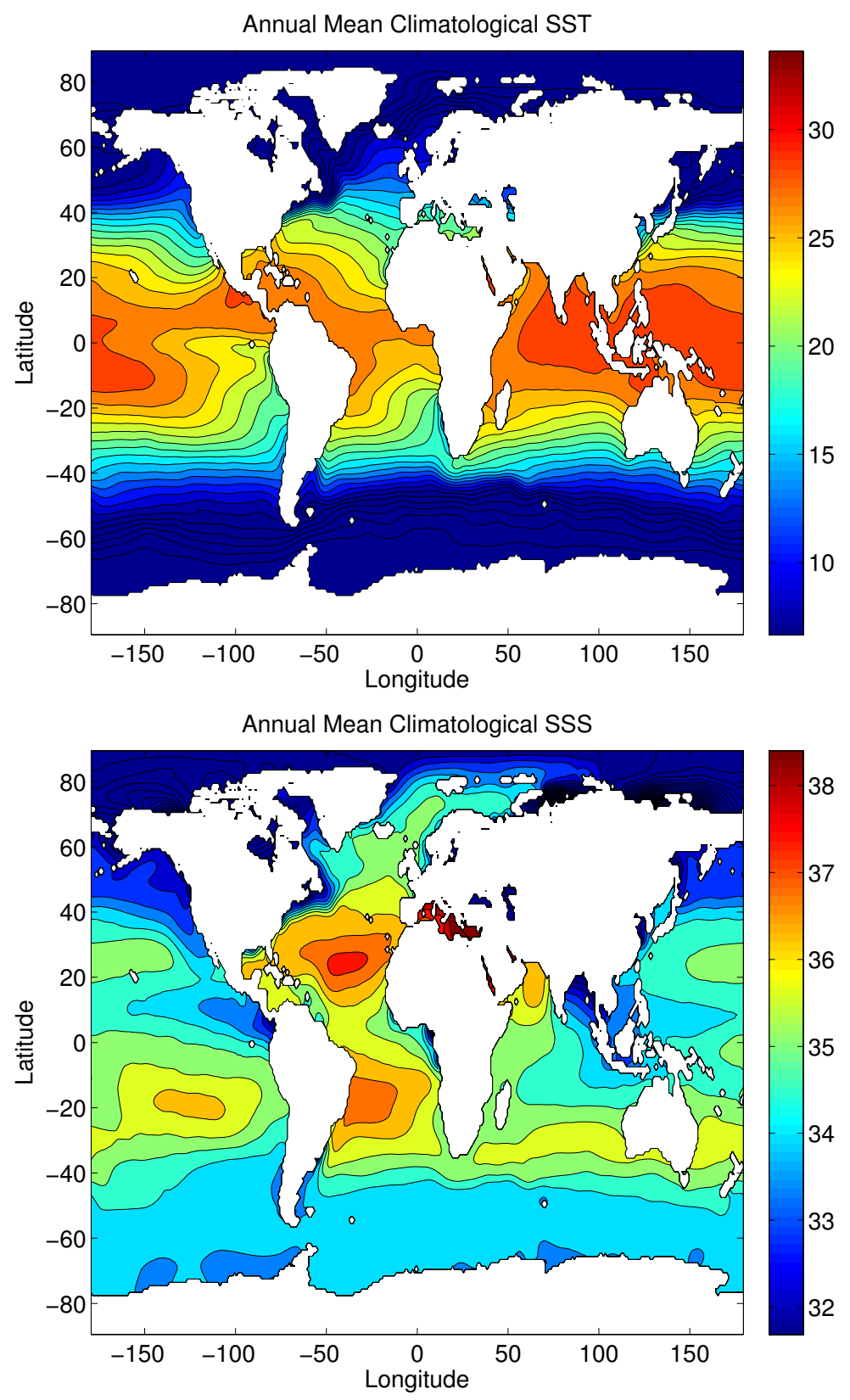

Figure 3: Climatological annual mean sea surface temperature (top panel, in degrees Celsius) and sea surface salinity (bottom panel, in $\mathrm{g} / \mathrm{kg}$ ) from the World Ocean Database 2013. 



Figure 4: (Top panel) Specific heat capacity $c_{p}$ (in $\mathrm{J} . \mathrm{K}^{-1} \cdot \mathrm{kg}^{-1}$ ) as a function of temperature and salinity at mean atmospheric pressure, illustrating the strong dependence of $c_{p}$ upon salinity. (Bottom panel) Surface distribution of $c_{p}$ (same units) for the climatological annual mean temperature and salinity fields depicted in Fig. 3. 

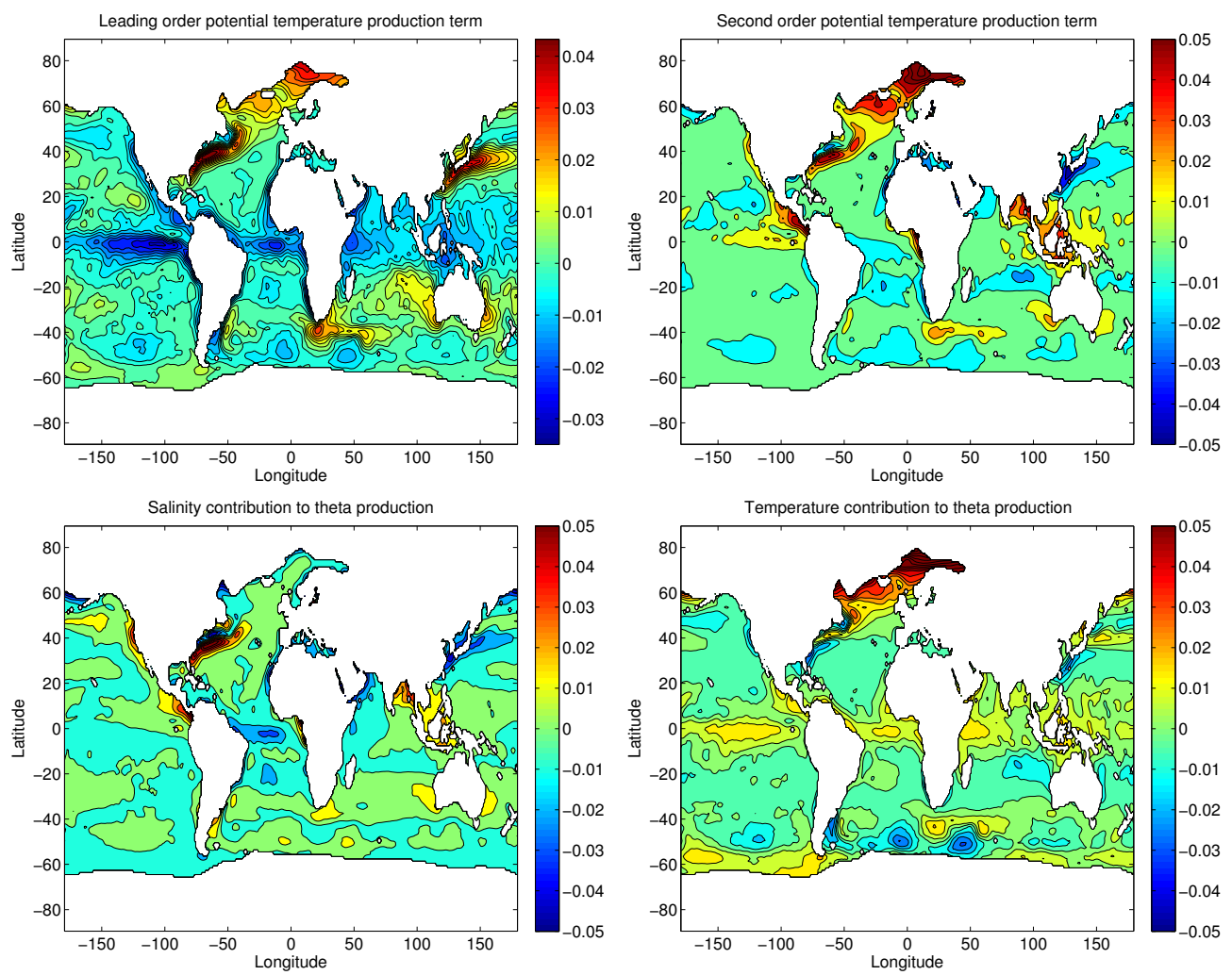

Figure 5: Leading order production term $-\tilde{Q} / \bar{c}_{p}^{R}$ (top left panel, in $\mathrm{kg} \cdot \mathrm{m}^{-2} \cdot \mathrm{s}^{-1} \cdot \mathrm{K}$ ) and second order production term $-\tilde{Q} /\left(1 / c_{p}^{R}-1 / \bar{c}_{p}^{R}\right)$ (top right panel, in $10^{-3} \mathrm{~kg} \cdot \mathrm{m}^{-2} \cdot \mathrm{s}^{-1} \cdot \mathrm{K}$ ). Decomposition of the top right panel into a salinity anomaly contribution (bottom left panel) and temperature anomaly contribution (bottom right panel), in same units as top right panel. 

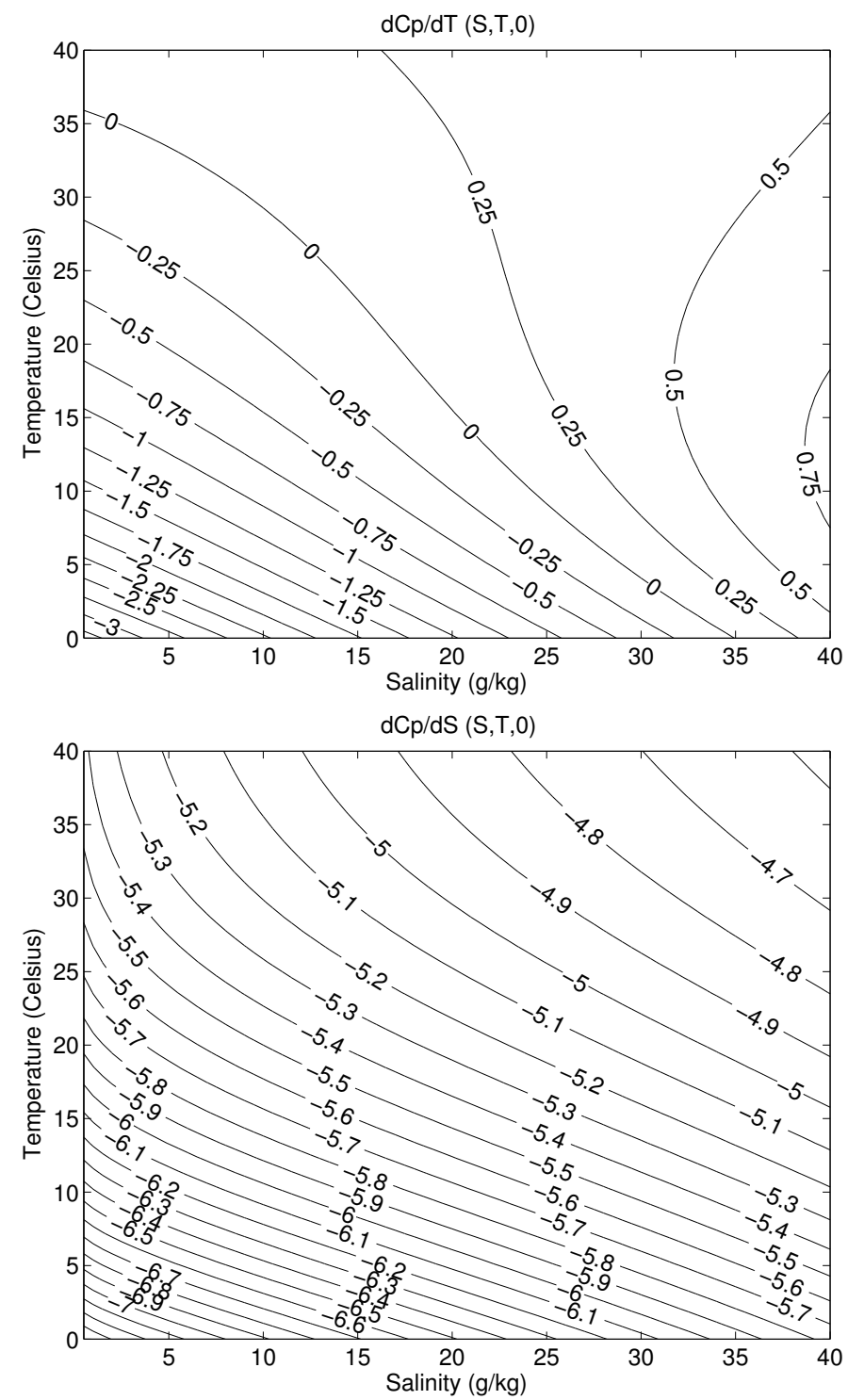

Figure 6: Derivative of the specific heat capacity with respect to temperature (top panel) and to salinity (bottom panel) as a function of temperature and salinity at mean atmospheric surface pressure. Units are respectively J.K ${ }^{-2} \cdot \mathrm{kg}^{-1}$ and $J . \mathrm{K}^{-1} \cdot \mathrm{kg}^{-1} \cdot(\mathrm{g} / \mathrm{kg})^{-1}$. 